\title{
Robust Stabilization of MIMO Systems in Finite/Fixed Time
}

\author{
A. Polyakov*, D. Efimov, W. Perruquetti \\ Non-A team, Inria Lille-Nord Europe, Parc Scientifique de la Haute Borne, 40, avenue Halley, Bat. A, Park Plaza, \\ 59650 Villeneuve d'Ascq, France
}

\begin{abstract}
SUMMARY
The control design problem for finite-time and fixed-time stabilization of linear multi-input system with nonlinear uncertainties and disturbances is considered. The control design algorithm based on block decomposition and Implicit Lyapunov Function (ILF) technique is developed. The robustness properties of the obtained control laws with respect to matched and unmatched uncertainties and disturbances are studied. Procedures for tuning of control parameters are presented in the form of Linear Matrix Inequalities (LMI). Aspects of practical implementation of developed algorithms are discussed. Theoretical results are supported by numerical simulations. Copyright (c) 2014 John Wiley \& Sons, Ltd.
\end{abstract}

Received ...

KEY WORDS: Non-aymptotic stabilization; Implicit Lyapunov Function; Homogeneity

\section{INTRODUCTION}

The quality of any control algorithm is always estimated by different performance indexes such as robustness with respect to disturbances, time optimality of transient motions, energetic effectiveness, etc. So, theoretically the design of a "good" control law is a multi-objective optimization problem. The mentioned criteria frequently contradict to each other. For example, time optimal (bang-bang) control is not robust and vice versa. From practical point of view, an optimality criterion can be relaxed or simply replaced with an alternative one. For instance, instead of minimum time control design the papers $[1,2,3,4,5,6]$ study the problem of non-asymptotic stabilization, when the convergence time is not minimal, but surely bounded by some predefined value.

Finite-time stabilization [1], [4], [7] is important if the transition time of the control system has to be specified in advance, for example, control of walking robot in between two impacts or the robot catching a "flying" ball. Control algorithms for finite-time stabilization of a chain of integrators are presented in different papers [3], [8], [5]. However, the tuning of control parameters of the proposed algorithms is complicated. This paper develops the methods of robust non-asymptotic (finite-time and fixed-time) stabilization of linear multi-input systems. One of the advantages of the control algorithms to be proposed is simplicity of tuning of control parameters using the LMI technique.

Fixed-time stability [6] assumes that convergence time of a finite-time stable system is bounded by some fixed number independently of the initial condition. The fixed-time approach helps to design a control law, which prescribes a transition time independently of operation domain. In practice, when maximum magnitude of the input is bounded, this control guarantees fixed-time stability property only locally. However, in contrast to other techniques the variation of operation domain in this case does not require re-tuning of the control parameters in order to preserve the convergence time. This paper also shows that fixed-time stability provides additional robustness

$\overline{{ }^{*} \text { Correspondence }}$ to: andrey.polyakov@inria.fr 
properties to the closed-loop system. The property, which is called today by fixed-time stability, was initially discovered in the context of locally homogeneous systems [9]. The application of this ideas to fixed-time differentiator design are presented in [10].

Homogeneity is a very useful property for finite-time stability analysis of control systems [11], [12], [8], [13], [5]. Particularly, if an asymptotically stable system is homogeneous of negative degree, then it is finite-time stable. However, even if a structure of homogeneous control is defined, then anyway a procedure for selection of control parameters has to be developed in order to guarantee, at least, asymptotic stability of a closed-loop system. Tuning of the convergence time is also very important for practical applications.

Lyapunov function method is the main approach to stability analysis and nonlinear control design. In this paper Implicit Lyapunov Function (ILF) method [14, 15, 16, 17] is applied for robust finitetime and fixed-time stabilization of linear multi-input plants. The ILF with ellipsoidal level sets is introduced using weighted homogeneity approach [18], [19], [20]. The paper develops the new control algorithms, which guarantee finite-time and fixed-time stabilization of linear MIMO system and reject bounded matched and "vanishing" unmatched disturbances of a certain type. The LMI representation of stability conditions are provided for simplicity of tuning of the control parameters. Weighted homogeneity and local homogeneity of the closed-loop systems is proven in order to expand the known Input-to-State Stability (ISS) results [21] to the obtained control systems. The paper also presents the proofs of theorems on the high-order sliding mode control design using ILF approach, which were announced in [22].

The sampled-time realization of fixed-time control algorithms can be complicated due to possible instability of the classical Eurler discretization scheme [23]. The ILF approach allows us to overcome this difficulty. The implicit Lyapunov function analysis implies an implicit definition of the control law that requires a special algorithm of practical implementations. By this reason the paper discusses the sampled-time realization of ILF control for linear system that admits on-line variation of feedback gains. The robustness of the presented scheme of ILF-control implementation is proven for an arbitrary sampling step.

The paper is organized as follows. The next section introduces the problem statement and the basic assumptions. Section III considers some preliminaries such as finite-time stability, weighted homogeneity and ILF method for finite-time and fixed-time stability analysis. After that the control design algorithm is presented. It is realized through several steps: decomposition into block controllability form [24] and finite-time (or fixed-time) ILF control design. Robustness issues of the developed control scheme are also studied in this section. Section IV discusses aspects of practical implementation of ILF control algorithms. Finally, simulation results and concluding remarks are presented.

Notation: $\mathbb{R}$ is the set of real numbers; $\mathbb{R}_{+}=\{x \in \mathbb{R}: x>0\} ;\|x\|$ denotes the Euclidian norm of the vector $x \in \mathbb{R}^{n}$; range $(B)$ is the column space of the matrix $B \in \mathbb{R}^{n \times m} ; \operatorname{diag}\left\{\lambda_{1}, \ldots, \lambda_{n}\right\}$ is a diagonal matrix with elements $\lambda_{i}$; the order relation $P>0(<0, \geq 0, \leq 0)$ for $P \in \mathbb{R}^{n \times n}$ means that $P$ is symmetric and positive (negative) definite (semidefinite); if $P>0$ then the matrix $P^{1 / 2}:=B$ is such that $B^{2}=P ; \lambda_{\max }(P)$ and $\lambda_{\min }(P)$ denote maximum and minimum eigenvalues of the symmetric matrix $P \in \mathbb{R}^{n \times n}$; a continuous function $\sigma: \mathbb{R}_{+} \rightarrow \mathbb{R}_{+}$belongs to the class $\mathcal{K}$ if it is monotone increasing and $\sigma(s) \rightarrow+0$ as $s \rightarrow+0$; $\operatorname{rown}(W)$ is the number of rows of a matrix $W$; $\operatorname{null}(W)$ denotes the matrix that has the columns defining an orthonormal basis of the null space of the matrix $W$.

\section{PROBLEM STATEMENT}

Consider the control system

$$
\dot{x}(t)=A x(t)+B u(t)+d(t, x(t)),
$$

where $x \in \mathbb{R}^{n}$ is the state vector, $u \in \mathbb{R}^{m}$ is the vector of control inputs, $A \in \mathbb{R}^{n \times n}$ is the system matrix, $B \in \mathbb{R}^{n \times m}$ is the matrix of control gains and the function $d: \mathbb{R} \times \mathbb{R}^{n} \rightarrow \mathbb{R}^{n}$ describes exogenous disturbances and uncertainties (e.g. uncertain nonlinearities of the system). 
It is assumed that the matrices $A$ and $B$ are known, $\operatorname{rank}(B)=m \leq n$ and the pair $(A, B)$ is controllable; the whole state vector $x$ can be measured and utilized for feedback control design. In order to include into consideration the case of discontinuous function $d$, Filippov theory of differential equations with discontinuous right-hand sides is applied.

The control aim is to stabilize the origin of the system (1) in a finite time or in a fixed time independently of the initial condition. In addition, the control has to reject disturbances of a certain type to be specified.

\section{PRELIMINARIES}

\subsection{Asymptotic Stability with Non-Asymptotic Transitions}

Consider the system of the form

$$
\dot{x}=f(t, x), \quad x(0)=x_{0},
$$

where $x \in \mathbb{R}^{n}$ is the state vector, $f: \mathbb{R}_{+} \times \mathbb{R}^{n} \rightarrow \mathbb{R}^{n}$ is a nonlinear vector field, which can be discontinuous with respect to the state variable. In this case the solutions $x\left(t, x_{0}\right)$ of the system (2) are understood in the sense of Filippov.

According to Filippov definition [25] an absolutely continuous function $x\left(t, x_{0}\right)$ is called a solution to the Cauchy problem associated to (2) if $x\left(0, x_{0}\right)=x_{0}$ and it satisfies the following differential inclusion

$$
\dot{x} \in K[f](t, x)=\bigcap_{\delta>0} \bigcap_{\mu(N)=0} \operatorname{co} f(t, x+B(\delta) \backslash N),
$$

where $\operatorname{co}(N)$ defines the convex closure of the set $N \subset \mathbb{R}^{n}$ and the equality $\mu(N)=0$ means that the set $N$ has measure 0 .

Assume that the origin is an equilibrium point of the system (2), i.e. $0 \in K[f](t, 0)$ for all $t \in \mathbb{R}$. The paper studies strong uniform stability properties of the system (2). The words "strong uniform" will be omitted below for shortness and simplicity of the presentation.

Definition 1 ([1], [4], [26])

The origin of system (2) is said to be globally finite-time stable if:

1. Finite-time attractivity: there exists a locally bounded function $T: \mathbb{R}^{n} \backslash\{0\} \rightarrow \mathbb{R}_{+}$, such that for all $x_{0} \in \mathbb{R}^{n} \backslash\{0\}$, any solution $x\left(t, x_{0}\right)$ of the system (2) is defined at least on $\left[0, T\left(x_{0}\right)\right)$ and $\lim _{t \rightarrow T\left(x_{0}\right)} x\left(t, x_{0}\right)=0$.

2. Lyapunov stability: $\exists \delta \in \mathcal{K}$ such that $\left\|x\left(t, x_{0}\right)\right\| \leq \delta\left(\left\|x_{0}\right\|\right)$ for all $x_{0} \in \mathbb{R}^{n}, t \in \mathbb{R}_{+}$.

The function $T$ is called the settling-time function of the system (2).

Definition 2 ([6])

The origin of system (2) is said to be globally fixed-time stable if it is globally finite-time stable and the settling-time function $T\left(x_{0}\right)$ is bounded, i.e. $\exists T_{\max } \in \mathbb{R}_{+}: T\left(x_{0}\right) \leq T_{\max }, \forall x_{0} \in \mathbb{R}^{n}$.

It is worth to stress that the finite-time or fixed-time stability always implies the asymptotic one.

\subsection{Homogeneity and Local Homogeneity}

Homogeneity [18], [11], [27], [19] is an intrinsic property of an object, which remains consistent with respect to some scaling: level sets (resp. solutions) are preserved for homogeneous functions (resp. vector fields).

Let $\lambda>0, r_{i}>0, i \in\{1, \ldots, n\}$ then one can define the vector of weights $r=\left(r_{1}, \ldots, r_{n}\right)^{T}$ and the dilation matrix $D(\lambda)=\operatorname{diag}\left\{\lambda^{r_{i}}\right\}_{i=1}^{n}$, where $\lambda \in \mathbb{R}_{+}$. Note that for $x=\left(x_{1}, \ldots, x_{n}\right)^{T} \in \mathbb{R}^{n}$ we have $D(\lambda) x=\left(\lambda^{r_{1}} x_{1}, \ldots, \lambda^{r_{i}} x_{i}, \ldots, \lambda^{r_{n}} x_{n}\right)^{T}$. 
Definition 3 ([18])

A function $g: \mathbb{R}^{n} \rightarrow \mathbb{R}$ (resp. a vector field $f: \mathbb{R}^{n} \rightarrow \mathbb{R}^{n}$ ) is said to be $r$-homogeneous of degree $m$ iff for all $\lambda>0$ and for all $x \in \mathbb{R}^{n}$ we have $g(D(\lambda) x)=\lambda^{m} g(x)$ (resp. $f(D(\lambda) x)=\lambda^{m} D(\lambda) f(x)$ ).

Theorem 4 ([12], Theorem 5.8 and Corollary 5.4)

Let $f: \mathbb{R}^{n} \rightarrow \mathbb{R}^{n}$ be defined on $\mathbb{R}^{n}$ and be a continuous $\mathbf{r}$-homogeneous vector field with a negative degree. If the origin of the system

$$
\dot{x}=f(x)
$$

is locally asymptotically stable then it is globally finite-time stable.

This theorem remains true for homogeneous differential inclusions [26, 8, 28].

The $r$-homogeneity property used in Definition 3 is introduced for some $r>0$ and all $\lambda \in \mathbb{R}_{+}$. Restricting the set of admissible values for $\lambda$, the local homogeneity [18], [9], [21] can be introduced. Let us introduce the following compact set (homogeneous sphere)

$$
S_{r}=\left\{x \in \mathbb{R}^{n}:\|x\|_{r}=1\right\},
$$

where $\|\cdot\|_{r}$ represents the $r$-homogeneous norm of $x \in \mathbb{R}^{n}$ defined by:

$$
\|x\|_{r}=\left(\left|x_{1}\right|^{\frac{\rho}{r_{1}}}+\ldots+\left|x_{i}\right|^{\frac{\rho}{r_{i}}}+\ldots+\left|x_{n}\right|^{\frac{\rho}{r_{n}}}\right)^{\frac{1}{\rho}}
$$

for some $\rho>0$.

Definition 5 ([21])

The function $g: \mathbb{R}^{n} \rightarrow \mathbb{R}, g(0)=0$ is called $\left(r, \lambda_{0}, g_{0}\right)$-homogeneous $\left(r \in \mathbb{R}_{+}^{n}, g_{0}: \mathbb{R}^{n} \rightarrow \mathbb{R}\right)$ if $g_{0}$ is $r$-homogeneous and $\lim _{\lambda \rightarrow \lambda_{0}} \lambda^{-d_{0}} g(D(\lambda) x)=g_{0}(x)$ for some $d_{0} \geq 0$ and any $x \in S_{r}$.

The vector field $f: \mathbb{R}^{n} \rightarrow \mathbb{R}^{n}$ is called $\left(r, \lambda_{0}, f_{0}\right)$-homogeneous $\left(r \in \mathbb{R}_{+}^{n}, f_{0}: \mathbb{R}^{n} \rightarrow \mathbb{R}^{n}\right)$ if $f_{0}$ is $r$ homogeneous and $\lim _{\lambda \rightarrow \lambda_{0}} \lambda^{-d_{0}} D^{-1}(\lambda) f(D(\lambda) x)=f_{0}(x)$ for some $d_{0} \geq-\min _{1 \leq i \leq n} r_{i}$ and any $x \in S_{r}$. The uniform convergence of the above limits is assumed for $\lambda_{0} \in\{0,+\infty\}$.

In [9] this definition was introduced for $\lambda_{0}=0$ and $\lambda_{0}=+\infty$ (the function $g$ is called homogeneous in the bi-limit if it is simultaneously $\left(r_{0}, 0, g_{0}\right)$-homogeneous and $\left(r_{\infty},+\infty, g_{\infty}\right)$ homogeneous), the case $\lambda_{0}=0$ has been also treated in $[29,20,12,3]$. The theorem below demonstrates the relation between fixed-time stability and homogeneity in bi-limit.

Theorem 6 ([9], Theorem 2.20 and Corollary 2.24)

Let the vector field $f: \mathbb{R}^{n} \rightarrow \mathbb{R}^{n}$ be $\left(r_{0}, 0, f_{0}\right)$-homogenous with degree $d_{0}<0$ and $\left(r_{\infty},+\infty, f_{\infty}\right)$-homogenous with degree $d_{\infty}>0$. If the system (4) and the systems $\dot{x}=f_{0}(x), \dot{x}=$ $f_{\infty}(x)$ are globally asymptotically stable, then the system (4) is globally fixed-time stable.

In addition to Theorems 4 and 6 the homogeneity theory provides many other advantages to analysis and design of nonlinear control system. For instance, some results about Input-to-State Stability of homogeneous systems can be found in [30, 31, 21].

\subsection{Implicit Lyapunov Function Method}

The next two theorem present recent extensions of the ILF method to finite-time and fixed-time stability analysis [17].

\section{Theorem 7}

If there exists a continuous function $Q: \mathbb{R}_{+} \times \mathbb{R}^{n} \rightarrow \mathbb{R}$ that satisfies the conditions

C1) $Q$ is continuously differentiable in $\mathbb{R}_{+} \times \mathbb{R}^{n} \backslash\{0\}$;

$C 2)$ for any $x \in \mathbb{R}^{n} \backslash\{0\}$ there exist $V \in \mathbb{R}_{+}$such that $Q(V, x)=0$;

$C 3)$ let $\Omega=\left\{(V, x) \in \mathbb{R}_{+} \times \mathbb{R}^{n}: Q(V, x)=0\right\}$ and

$$
\lim _{\substack{x \rightarrow 0 \\(V, x) \in \Omega}} V=0^{+}, \lim _{\substack{V \rightarrow 0^{+} \\(V, x) \in \Omega}}\|x\|=0, \lim _{\substack{\|x\| \rightarrow \infty \\(V, x) \in \Omega}} V=+\infty ;
$$


C4) the inequality $\frac{\partial Q(V, x)}{\partial V}<0$ holds for all $V \in \mathbb{R}_{+}$and $x \in \mathbb{R}^{n} \backslash\{0\}$;

C5) there exist $c \in \mathbb{R}_{+}$and $\mu \in(0,1]$ such that

$$
\sup _{t \in \mathbb{R}_{+}, y \in K[f](t, x)} \frac{\partial Q(V, x)}{\partial x} y \leq c V^{1-\mu} \frac{\partial Q(V, x)}{\partial V}, \quad(V, x) \in \Omega
$$

then the origin of system (2) is globally finite time stable with the following settling time estimate: $T\left(x_{0}\right) \leq \frac{V_{0}^{\mu}}{c \mu}$, where $V_{0} \in \mathbb{R}_{+}: Q\left(V_{0}, x_{0}\right)=0$.

Theorem 7 represents the well-known stability result on finite-time stability (see, for example, [4]) for implicit definition of Lyapunov function. Indeed, the conditions $C 1), C 2$ ) and C4) guarantee existence and uniqueness of a positive definite function $V: \mathbb{R}^{n} \rightarrow \mathbb{R}_{+}$such that $Q(V(x), x)=0$ for all $x \in \mathbb{R}^{n}$. The conditions $C 3$ ) implies that $V(x) \rightarrow 0$ as $x \rightarrow 0$ and $V(x) \rightarrow+\infty$ as $x \rightarrow \infty$. The Implicit Function Theorem [32] provides the formula for the partial derivative $\frac{\partial V}{\partial x}=-\left[\frac{\partial Q}{\partial V}\right]^{-1} \frac{\partial Q}{\partial x}$. Hence, the conditions $C 4$ ) and $C 5$ ) give the estimate of the time derivative $\dot{V}(x) \leq-c V^{1-\mu}$ implying the finite-time stability [4].

Theorem 8

Let there exist two functions $Q_{1}: \mathbb{R}_{+} \times \mathbb{R}^{n} \rightarrow \mathbb{R}$ and $Q_{2}: \mathbb{R}_{+} \times \mathbb{R}^{n} \rightarrow \mathbb{R}$ that satisfy the conditions C1)-C4) of Theorem 7 and

C6) $Q_{1}(1, x)=Q_{2}(1, x)$ for all $x \in \mathbb{R}^{n} \backslash\{0\}$;

C7) there exist $c_{1} \in \mathbb{R}_{+}$and $\mu \in(0,1]$ such that

$$
\sup _{t \in \mathbb{R}_{+}, y \in K[f](t, x)} \frac{\partial Q_{1}(V, x)}{\partial x} y \leq c_{1} V^{1-\mu} \frac{\partial Q_{1}(V, x)}{\partial V}
$$

for all $V \in(0,1]$ and $x \in \mathbb{R}^{n} \backslash\{0\}$ satisfying $Q_{1}(V, x)=0$;

C8) there exist $c_{2} \in \mathbb{R}_{+}$and $\nu \in \mathbb{R}_{+}$such that

$$
\sup _{t \in \mathbb{R}_{+}, y \in K[f](t, x)} \frac{\partial Q_{2}(V, x)}{\partial x} y \leq c_{2} V^{1+\nu} \frac{\partial Q_{2}(V, x)}{\partial V}
$$

for all $V \geq 1$ and $x \in \mathbb{R}^{n} \backslash\{0\}$ satisfying $Q_{2}(V, x)=0$;

Then the equilibrium point $x=0$ of the system (2) is globally fixed-time stable with the global estimate of the settling time: $T\left(x_{0}\right) \leq \frac{1}{c_{1} \mu}+\frac{1}{c_{2} \nu}$.

Below we use these theorems for the finite-time and fixed-time stabilizing feedbacks design.

\section{CONTROL DESIGN USING IMPLICIT LYAPUNOV FUNCTION METHOD}

\subsection{Block Decomposition}

Let us initially decompose the original multi-input system (1) to a block form [24]. Below we use the known block decomposition procedure discussed in $[6,22]$. Due to this reason many details are skipped for simplicity of the presentation.

Let the orthogonal matrices $T_{i}$ be defined by the following two step algorithm:

Initialization : $A_{0}=A, B_{0}=B, T_{0}=I_{n}, k=0$.

Loop: While $\operatorname{rank}\left(B_{k}\right)<\operatorname{rown}\left(A_{k}\right)$ do

$$
A_{k+1}=B_{k}^{\perp} A_{k}\left(B_{k}^{\perp}\right)^{T}, \quad B_{k+1}=B_{k}^{\perp} A_{k} \hat{B}_{k}, \quad T_{k+1}=\left(\begin{array}{c}
B_{k}^{\perp} \\
\hat{B}_{k}
\end{array}\right), \quad k=k+1,
$$

where $B_{k}^{\perp}:=\left(\operatorname{null}\left(B_{k}^{T}\right)\right)^{T}, \hat{B}_{k}:=\left(\operatorname{null}\left(B_{k}^{\perp}\right)\right)^{T}$.

In the paper [6] it was proven that the orthogonal matrix

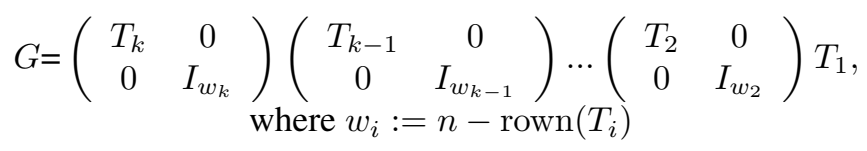


provides

$$
\begin{aligned}
G A G^{T}= & \left(\begin{array}{ccccc}
A_{11} & A_{12} & 0 & \ldots & 0 \\
A_{21} & A_{22} & A_{23} & \ldots & 0 \\
\ldots & \ldots & \ldots & \ldots & \ldots \\
A_{k-11} & A_{k-12} & \ldots & A_{k-1 k-1} & A_{k-1 k} \\
A_{k 1} & A_{k 2} & \ldots & A_{k k-1} & A_{k k}
\end{array}\right), \\
& G B=\left(\begin{array}{lllll}
0 & 0 & \ldots & 0 & A_{k k+1}^{T}
\end{array}\right)^{T},
\end{aligned}
$$

where $A_{k k+1}=\hat{B}_{0} B_{0}, A_{i j} \in \mathbb{R}^{n_{i} \times n_{j}}, n_{i}:=\operatorname{rank}\left(B_{k-i}\right), i, j=1,2, \ldots, k$ and $\operatorname{rank}\left(A_{i i+1}\right)=n_{i}$.

Recall that the $B$ has full column $\operatorname{rank}(\operatorname{rank}(B)=m)$. Consequently, $A_{k k+1}$ is square and nonsingular. Since $\operatorname{rank}\left(A_{i i+1}\right)=n_{i}=\operatorname{rown}\left(A_{i i+1}\right)$ then $A_{i i+1} A_{i i+1}^{T}$ is invertible and $A_{i i+1}^{+}=$ $A_{i+1}^{T}\left(A_{i+1} A_{i i+1}^{T}\right)^{-1}$ is the right inverse matrix of $A_{i i+1}$. Introduce the linear coordinate transformation $s=\Phi y, s=\left(s_{1}, \ldots, s_{k}\right)^{T}, s_{i} \in \mathbb{R}^{n_{i}}, y=\left(y_{1}, \ldots, y_{k}\right)^{T}, y_{i} \in \mathbb{R}^{n_{i}}$ by the formulas:

$$
\begin{gathered}
s_{i}=y_{i}+\varphi_{i}, \quad i=1,2, \ldots, k \\
\varphi_{1}=0, \quad \varphi_{i+1}=A_{i i+1}^{+}\left(\sum_{j=1}^{i} A_{i j} y_{j}+\sum_{r=1}^{i} \frac{\partial \varphi_{i}}{\partial y_{r}} \sum_{j=1}^{r+1} A_{r j} y_{j}\right) .
\end{gathered}
$$

The presented coordinate transformation is linear and nonsingular. The inverse transformation $y=\Phi^{-1} s$ is defined as follows:

$$
\begin{gathered}
y_{i}=s_{i}+\psi_{i}, \quad i=1,2, \ldots, k \\
\psi_{1}=0, \quad \psi_{i+1}=A_{i i+1}^{+}\left(\sum_{k=1}^{i} \frac{\partial \psi_{i}}{\partial s_{k}} A_{i i+1} s_{k+1}-\sum_{j=1}^{i} A_{i j}\left(s_{j}+\psi_{j}\right)\right) .
\end{gathered}
$$

For example, if $k=3$ then the matrix $\Phi$ has the form

$$
\Phi=\left(\begin{array}{ccc}
I_{n_{1}} & 0 & 0 \\
A_{12}^{+} A_{11} & I_{n_{2}} & 0 \\
A_{23}^{+}\left(A_{21}+A_{12}^{+} A_{11}^{2}\right) & A_{23}^{+}\left(A_{22}+A_{12}^{+} A_{11} A_{12}\right) & I_{n_{3}}
\end{array}\right) .
$$

In general case, the transformation $\Phi$ can be calculated numerically in MATLAB.

Applying the transformation $s=\Phi G x$ to the system (1) we obtain

$$
\dot{s}=\left(\begin{array}{ccccc}
0 & A_{12} & 0 & \ldots & 0 \\
0 & 0 & A_{23} & \ldots & 0 \\
\ldots & \ldots & \ldots & \ldots & \ldots \\
0 & 0 & \ldots & 0 & A_{k-1 k} \\
\tilde{A}_{k 1} & \tilde{A}_{k 2} & \ldots & \tilde{A}_{k k-1} & \tilde{A}_{k k}
\end{array}\right) s+B^{\prime} u+\tilde{d}
$$

where the block $\tilde{A}_{k i}$ has the same size as $A_{k i}, B^{\prime}=\Phi G B=\left(\begin{array}{ccccc}0 & 0 & \ldots & 0 & A_{k k+1}^{T}\end{array}\right)^{T}$ and

$$
\tilde{d}:=\tilde{d}(t, s)=\Phi G d\left(t, G^{T} \Phi^{-1} s\right) .
$$

Let us select the control law in the form

$$
u=A_{k k+1}^{+}\left(\tilde{u}-K_{l i n} s\right),
$$

where $K_{\text {lin }}=\left(\begin{array}{lllll}\tilde{A}_{k 1} & \tilde{A}_{k 2} & \ldots & \tilde{A}_{k k-1} & \tilde{A}_{k k}\end{array}\right)$ and $\tilde{u} \in \mathbb{R}^{n_{k}}$ is a nonlinear part of feedback, which has to be designed in order to guarantee finite-time stability of the origin of the system:

$$
\dot{s}=\tilde{A} s+\tilde{B} \tilde{u}+\tilde{d}(t, s),
$$


where

$$
\tilde{A}=\left(\begin{array}{ccccc}
0 & A_{12} & 0 & \ldots & 0 \\
0 & 0 & A_{23} & \ldots & 0 \\
\ldots & \ldots & \ldots & \ldots & \ldots \\
0 & \ldots & \ldots & 0 & A_{k-1 k} \\
0 & \ldots & \ldots & 0 & 0
\end{array}\right), \quad \tilde{B}=\left(\begin{array}{ccccc}
0 & 0 & \ldots & 0 & I_{n_{k}}
\end{array}\right)^{T} \in \mathbb{R}^{n \times n_{k}}
$$

\section{Remark 9}

Feedback linearizable nonlinear systems $\dot{x}=f(x)+G(x) u$ can also be transformed into the form (12) (see, for example, [33]).

\subsection{Finite-time Stabilization}

Introduce the ILF function

$$
Q(V, s):=s^{T} D_{r}\left(V^{-1}\right) P D_{r}\left(V^{-1}\right) s-1,
$$

where $s=\left(s_{1}, \ldots, s_{k}\right)^{T}, s_{i} \in \mathbb{R}^{n_{i}}, V \in \mathbb{R}^{+}, D_{r}(\lambda)$ is the dilation matrix of the form

$$
\begin{aligned}
D_{r}(\lambda)= & \left(\begin{array}{cccc}
\lambda^{r_{1}} I_{n_{1}} & 0 & \ldots & 0 \\
0 & \lambda^{r_{2}} I_{n_{2}} & \ldots & 0 \\
\ldots & \cdots & \cdots & \ldots \\
0 & \cdots & 0 & \lambda^{r_{k}} I_{n_{k}}
\end{array}\right), \quad \lambda \in \mathbb{R}_{+}, \\
& r_{i}=1+(k-i) \mu, \quad i=1,2, . ., k,
\end{aligned}
$$

$0<\mu \leq 1$ and $P \in \mathbb{R}^{n \times n}$ is a symmetric positive definite matrix, i.e. $P=P^{T}>0$. Denote $H_{\mu}:=$ $\operatorname{diag}\left\{r_{i} I_{n_{i}}\right\}_{i=1}^{k}$.

\section{Theorem 10 (On finite-time stabilization without perturbations)}

If $\tilde{d}(t, s) \equiv 0$ and the system of matrix inequalities:

$$
\left\{\begin{array}{c}
\tilde{A} X+X \tilde{A}^{T}+\tilde{B} Y+Y^{T} \tilde{B}^{T}+H_{\mu} X+X H_{\mu}=0 \\
X H_{\mu}+H_{\mu} X>0, \quad X>0
\end{array},\right.
$$

is feasible for some $\mu \in(0,1]$ and $X \in \mathbb{R}^{n \times n}, Y \in \mathbb{R}^{n_{k} \times n}$ then the control of the form (11) with

$$
\tilde{u}=\tilde{u}(V, s)=V^{1-\mu} K D_{r}\left(V^{-1}\right) s,
$$

where $K:=Y X^{-1}, V \in \mathbb{R}_{+}$satisfies $Q(V, s)=0$ and $Q$ is defined by (14) with $P:=X^{-1}$, stabilizes the origin of the system (1) in a finite time and the settling-time function is defined by

$$
T\left(x_{0}\right)=\frac{V_{0}^{\mu}}{\mu},
$$

where $V_{0} \in \mathbb{R}_{+}: Q\left(V_{0}, \Phi G x_{0}\right)=0$.

Proof

The function $Q(V, s)$ defined by (14) satisfies the conditions $C 1)-C 3$ ) of Theorem 7. Indeed, it is continuously differentiable for all $V \in \mathbb{R}_{+}$and $\forall s \in \mathbb{R}^{n}$. Since $P>0$ then the following chain of inequalities

$$
\frac{\lambda_{\min }(P)\|s\|^{2}}{\max \left\{V^{2+2(k-1) \mu}, V^{2}\right\}} \leq Q(V, s)+1 \leq \frac{\lambda_{\max }(P)\|s\|^{2}}{\min \left\{V^{2+2(k-1) \mu}, V^{2}\right\}}
$$

implies that for any $s \in \mathbb{R}^{n} \backslash\{0\}$ there exist $V^{-} \in \mathbb{R}_{+}$and $V^{+} \in \mathbb{R}_{+}: Q\left(V^{-}, s\right)<0<Q\left(V^{+}, s\right)$. Moreover, if $Q(V, s)=0$ then the same chain of inequalities gives

$$
\frac{\min \left\{V^{2+2(k-1) \mu}, V^{2}\right\}}{\lambda_{\max }(P)} \leq\|s\|^{2} \leq \frac{\max \left\{V^{2+2(k-1) \mu}, V^{2}\right\}}{\lambda_{\min }(P)} .
$$


It follows that the condition $C 3$ ) of Theorem 7 holds.

Since

$$
\frac{\partial Q}{\partial V}=-V^{-1} s^{T} D_{r}\left(V^{-1}\right)\left(H_{\mu} P+P H_{\mu}\right) D_{r}\left(V^{-1}\right) s,
$$

then (16) and $P:=X^{-1}$ implies $H_{\mu} P+P H_{\mu}>0$ and $\frac{\partial Q}{\partial V}<0$ for $\forall V \in \mathbb{R}_{+}$and $s \in \mathbb{R}^{n} \backslash\{0\}$. So the condition $C 4$ ) of Theorem 7 also holds. In this case we have

$$
\frac{\partial Q}{\partial s}(\tilde{A} s+\tilde{B} \tilde{u})=2 s^{T} D_{r}\left(V^{-1}\right) P D_{r}\left(V^{-1}\right)(\tilde{A} s+\tilde{B} \tilde{u}) .
$$

Taking into account that $D_{r}\left(V^{-1}\right) \tilde{A} D_{r}^{-1}\left(V^{-1}\right)=V^{-\mu} \tilde{A}$ and $D_{r}\left(V^{-1}\right) \tilde{B} \tilde{u}=V^{-\mu} \tilde{B} K D_{r}\left(V^{-1}\right) s$ we obtain

$$
\left.\frac{\partial Q}{\partial s}(\tilde{A} s+\tilde{B} \tilde{u})\right)=V^{-\mu} s^{T} D_{r}\left(V^{-1}\right)\left(P(\tilde{A}+\tilde{B} K)+(\tilde{A}+\tilde{B} K)^{T} P\right) D_{r}\left(V^{-1}\right) s .
$$

Therefore,

$$
\begin{gathered}
\dot{V}=-\left[\frac{\partial Q}{\partial V}\right]^{-1} \frac{\partial Q}{\partial s}(\tilde{A} s+\tilde{B} \tilde{u})=\frac{s^{T} D_{r}\left(V^{-1}\right)\left(P(\tilde{A}+\tilde{B} K)+(\tilde{A}+\tilde{B} K)^{T} P\right) D_{r}\left(V^{-1}\right) s}{s^{T} D_{r}\left(V^{-1}\right)\left(H_{\mu} P+P H_{\mu}\right) D_{r}\left(V^{-1}\right) s} V^{1-\mu}= \\
\frac{z^{T}\left(P(\tilde{A}+\tilde{B} K)+(\tilde{A}+\tilde{B} K)^{T} P\right) z}{z^{T}\left(H_{\mu} P+P H_{\mu}\right) z} V^{1-\mu}=-V^{1-\mu},
\end{gathered}
$$

where $z:=z(V, s)=D_{r}\left(V^{-1}\right) s$ and the equality from (16) was used on the last step.

\section{Remark 11}

The practical implementation of the control (17) requires solving of the equation $Q(V, s)=0$ in order to obtain $V(s)$. In some cases (for example, $k=2, \mu=1$ ), the function $V(s)$ can be found analytically. In other cases this equation can be solved numerically and on-line during digital implementation of a control law. A more detailed study of the practical implementation of the ILF control algorithm is presented in Section 5.

The system of matrix inequalities (16) can be easily solved using LMI toolbox of MATLAB or, for example, SeDuMi solver. The solution of (16) also can be constructed analytically using the proof of the next proposition.

Proposition 12

The system of matrix inequalities (16) is feasible for any $\mu \in \mathbb{R}_{+}$.

Proof

Let us represent the matrices $X, Y$ in the block form

$$
\begin{gathered}
X=\left(\begin{array}{ccccc}
X_{11} & X_{12} & \ldots & X_{1 k-1} & X_{1 k} \\
X_{12}^{T} & X_{22} & \ldots & X_{2 k-1} & X_{2 k-1} \\
\ldots & \ldots & \ldots & \ldots & \ldots \\
X_{1 k-1}^{T} & X_{2 k-1}^{T} & \ldots & X_{k-1 k-1} & X_{k-1 k} \\
X_{1 k}^{T} & X_{1 k-1}^{T} & \ldots & X_{k-1 k}^{T} & X_{k k}
\end{array}\right), X_{i j} \in \mathbb{R}^{n_{i} \times n_{j}}, i, j=1,2, \ldots, k ; \\
Y=\left(\begin{array}{ccccc}
Y_{1} & Y_{2} & \ldots & Y_{k-1} & Y_{k}
\end{array}\right), Y_{i} \in \mathbb{R}^{n_{k} \times n_{i}}, i=1,2, \ldots, k .
\end{gathered}
$$

The algebraic equation from (16) can be equivalently rewritten in the block form

$$
\begin{aligned}
A_{i i+1} X_{i i+1}^{T}+X_{i i+1} A_{i i+1}^{T}+2[1+\mu(k-i)] X_{i i}=0, & i=1,2, \ldots, k-1, \\
A_{i i+1} X_{i+1 j}+X_{i j+1} A_{j j+1}^{T}+[2+\mu(2 k-i-j)] X_{i j}=0, & j>i=1,2, \ldots, k-1, \\
A_{i i+1} X_{i+1 k}+[2+\mu(k-i)] X_{i k}+Y_{i}^{T}=0, & i=1,2, \ldots, k-1, \\
2 X_{k k}+Y_{k}^{T}+Y_{k}=0 . &
\end{aligned}
$$


Let $\mathbf{X}_{\left(i_{1}: i_{2} ; j_{1}: j_{2}\right)}$ be the block matrix consisting of the blocks $X_{i j}$ with $i=i_{1}, i_{1}+1, \ldots, i_{2}$ and $j=j_{1}, j_{1}+1, \ldots, j_{2}$, where $i_{1} \leq i_{2}$ and $j_{1} \leq j_{2}$. Denote $H_{i}=\operatorname{diag}\left\{(1+\mu k-\mu) I_{n_{1}},(1+\mu k-\right.$ $\left.2 \mu) I_{n_{2}}, \ldots,(1+\mu k-i \mu) I_{n_{i}}\right\}$ and $Z=X H_{\mu}+H_{\mu} X$. Let $\mathbf{Z}_{\left(i_{1}: i_{2} ; j_{1}: j_{2}\right)}$ be the block matrix of the same structure like $\mathbf{X}_{\left(i_{1}: i_{2} ; j_{1}: j_{2}\right)}$ but constructed for the matrix $Z$. Evidently, we have

$$
\mathbf{Z}_{(1: 1 ; 1: 1)}=2[1+\mu(k-1)] X_{(1: 1 ; 1: 1)}, \quad \mathbf{Z}_{(1: i ; 1: i)}=\left(\begin{array}{cc}
\mathbf{Z}_{(1: i-1 ; 1: i-1)} & \mathbf{X}_{(1: i-1 ; i: i)} H_{i} \\
\mathbf{X}_{(1: i-1 ; i: i)}^{T} H_{i} & 2[1+\mu(k-i)] X_{i i}
\end{array}\right) .
$$

Let us construct by induction the solution of the system (19)-(22) such that $X>0$ and $Z>0$. The next considerations use the property $\operatorname{rank}\left(A_{i i+1}\right)=n_{i}$.

Induction base. Select $X_{11}=\alpha_{1} I_{n_{1}}$, where $\alpha_{1}>0$ is an arbitrary positive number. In this case the equation (19) gives $X_{12}=-\alpha_{1}[1+\mu(k-1)] X_{11} A_{12}^{+} A_{12}$. Since $X_{11}=\mathbf{X}_{(1: 1 ; 1: 1)}>0$ and $\mathbf{Z}_{(1: 1 ; 1: 1)}>0$ then selecting $X_{22}=\alpha_{2} I_{n_{2}}$ we have $\mathbf{X}_{(1: 2 ; 1: 2)}>0$ and $\mathbf{Z}_{(1: 2 ; 1: 2)}>0$ for sufficiently large $\alpha_{2}>0$.

Induction step. Let for some $\tilde{k}<k$ the matrices $\mathbf{X}_{(1: \tilde{k} ; 1: \tilde{k})}>0$ and $\mathbf{Z}_{(1: \tilde{k} ; 1: \tilde{k})}>0$ be constructed such that $X_{i i}=\alpha_{i} I_{n_{i}}, \alpha_{i} \in \mathbb{R}_{+}$. The equation (19) gives $X_{\tilde{k} \tilde{k}+1}=-\alpha_{\tilde{k}}[1+\mu(k-$ $\tilde{k})] A_{\tilde{k} \tilde{k}+1}^{+} A_{\tilde{k} \tilde{k}+1}$ and the equation (20) implies

$$
X_{i \tilde{k}+1}=-\left(A_{i i+1} X_{i+1 \tilde{k}}\right) A_{\tilde{k} \tilde{k}+1}^{+} A_{\tilde{k} \tilde{k}+1}, \quad i=1,2 \ldots, \tilde{k} .
$$

Since $\mathbf{X}_{(1: \tilde{k} ; 1: \tilde{k})}>0$ and $\mathbf{Z}_{(1: \tilde{k} ; 1: \tilde{k})}>0$ then selecting $X_{\tilde{k}+1 \tilde{k}+1}=\alpha_{\tilde{k}+1} I_{n_{\tilde{k}+1}}$ we will have $\mathbf{X}_{(1: \tilde{k}+1 ; 1: \tilde{k}+1)}>0$ and $\mathbf{Z}_{(1: \tilde{k}+1 ; 1: \tilde{k}+1)}>0$ for sufficiently large $\alpha_{\tilde{k}+1}>0$.

The presented algorithm constructs $X>0$ such that $Z>0$ and the equations (19), (20) holds. On the last step (when $\tilde{k}=k$ ), selecting $Y_{k}=X_{k k}$ and

$$
Y_{i}=-\left(A_{i+1} X_{i+1 k}+[2+\mu(k-i)] X_{i k}\right)^{T}, \quad i=1,2, \ldots, k-1,
$$

we obtain $X>0$ and $Y$ satisfying (19)-(22) and the inequality $X H_{\mu}+H_{\mu} X>0$.

Note that the formula (18) provides the exact value of the settling-time.

\section{Remark 13}

For $\mu \in(0,1)$ the control of the form (17) is continuous function of the state $x$. If $\mu=1$ then the control function $\tilde{u}$ is continuous outside the origin and bounded for all $x \in \mathbb{R}^{n}$. Indeed, since $s^{T} D_{r}\left(V^{-1}\right) P D_{r}\left(V^{-1}\right) s=1 \Rightarrow\left\|D_{r}\left(V^{-1}\right) s\right\|^{2} \leq \frac{1}{\lambda_{\min }(P)}$ then for $\mu=1$ we have

$$
\tilde{u}^{2} \leq\|K\|^{2} \cdot\left\|D_{r}\left(V^{-1}\right) s\right\|^{2} \leq \frac{\|K\|^{2}}{\lambda_{\min }(P)} .
$$

Hence, it is easy to see that for $\mu=1$ in order to restrict the control magnitude by $\|\tilde{u}\| \leq u_{0}$ the following matrix inequality

$$
\left(\begin{array}{cc}
X & Y^{T} \\
Y & u_{0}^{2} I_{m}
\end{array}\right) \geq 0
$$

can be added to (16).

The control law (17) is Implicit Lyapunov Function-based control or shortly ILF control [17].

\section{Corollary 14}

If $\tilde{d} \equiv 0$ then the system (12), (17) is $r$-homogeneous of degree $-\mu$ with $r=(1+(k-1) \mu, 1+$ $(k-2) \mu, \ldots, 1)$. The Implicit Lyapunov Function $V(x)$ is $r$-homogeneous of degree 1 .

Proof

Obviously, we have $Q\left(V, D_{r}(\lambda) s\right)=Q\left(\lambda^{-1} V\right.$, s), i.e. $V\left(D_{r}(\lambda) s\right)=\lambda V(s)$. Now, we derive

$$
\begin{gathered}
\tilde{u}\left(D_{r}(\lambda) s\right)=V^{1-\mu}\left(D_{r}(\lambda) s\right) K D_{r}\left(V^{-1}\left(D_{r}(\lambda) s\right)\right) D_{r}(\lambda) s \\
=\lambda^{1-\mu} V^{1-\mu}(s) K D_{r}\left(\lambda^{-1} V^{-1}(s)\right) D_{r}(\lambda) s=\lambda^{1-\mu} \tilde{u}(x)
\end{gathered}
$$

and $\tilde{A} D_{r}(\lambda) s+\tilde{B} \tilde{u}\left(D_{r}(\lambda) s\right)=\lambda^{-\mu} D_{r}(\lambda)(\tilde{A} s+\tilde{B} \tilde{u}(s))$. 
The proven corollary transfers all qualitative robustness properties of homogeneous systems (like Input-to-State Stability) to the system (12), (17) (see, for example, [30, 31, 21]). In the same time, the control practice is mainly interested in quantitative analysis of robustness. The next theorem presents the modification of ILF control scheme rejecting some additive disturbances.

Theorem 15 (On finite-time robust stabilization)

If $\mu \in(0,1]$ and the system of linear matrix inequalities

$$
\left\{\begin{array}{c}
\tilde{A} X+X \tilde{A}^{T}+\tilde{B} Y+Y^{T} \tilde{B}^{T}+H_{\mu} X+X H_{\mu}+R \leq 0 \\
X H_{\mu}+H_{\mu} X>0, \quad X>0, \quad X \in \mathbb{R}^{n \times n}, \quad Y \in \mathbb{R}^{n_{k} \times n}
\end{array}\right.
$$

is feasible for some fixed $R \in \mathbb{R}^{n \times n}, R>0$ and the control $\tilde{u}=\tilde{u}(V, s)$ has the form (17) with $P:=X^{-1}$ and $K=Y X^{-1}$, then for any continuous disturbance function $\tilde{d}$ satisfying the inequality

$$
\begin{gathered}
\tilde{d}^{T} D_{r}\left(V^{-1}\right) R^{-1} D_{r}\left(V^{-1}\right) \tilde{d} \leq \beta V^{-2 \mu} s^{T} D_{r}\left(V^{-1}\right)\left(H_{\mu} P+P H_{\mu}\right) D_{r}\left(V^{-1}\right) s, \\
\text { with } V \in \mathbb{R}_{+}: Q(V, s)=0 \text { and } \beta \in(0,1)
\end{gathered}
$$

the closed-loop system (1), (11) is globally finite-time stable and the settling-time function estimate has the form

$$
T\left(x_{0}\right) \leq \frac{V_{0}^{\mu}}{(1-\beta) \mu}
$$

where $V_{0} \in \mathbb{R}_{+}: Q\left(V_{0}, \Phi G x_{0}\right)=0$ and $Q$ is defined by (14).

Proof

Taking into account the disturbances we obtain

$$
\begin{gathered}
\frac{\partial Q}{\partial s}(\tilde{A} s+\tilde{B} \tilde{u}+\tilde{d})=V^{-\mu} s^{T} D_{r}\left(V^{-1}\right)\left(P(\tilde{A}+\tilde{B} K)+(\tilde{A}+\tilde{B} K)^{T} P\right) D_{r}\left(V^{-1}\right) s+ \\
s^{T} D_{r}\left(V^{-1}\right) P D_{r}\left(V^{-1}\right) \tilde{d}+\tilde{d}^{T} D_{r}\left(V^{-1}\right) P D_{r}\left(V^{-1}\right) s=\left(\begin{array}{c}
D_{r}\left(V^{-1}\right) s \\
D_{r}\left(V^{-1}\right) \tilde{d}
\end{array}\right)^{T} W\left(\begin{array}{c}
D_{r}\left(V^{-1}\right) s \\
D_{r}\left(V^{-1}\right) \tilde{d}
\end{array}\right)+ \\
V^{\mu} \tilde{d}^{T} D_{r}\left(V^{-1}\right) R^{-1} D_{r}\left(V^{-1}\right) \tilde{d}-V^{-\mu} s^{T} D_{r}\left(V^{-1}\right)\left(H_{\mu} P+P H_{\mu}\right) D_{r}\left(V^{-1}\right) s,
\end{gathered}
$$

where

$$
W:=\left(\begin{array}{cc}
V^{-\mu}\left(P(\tilde{A}+\tilde{B} K)+(\tilde{A}+\tilde{B} K)^{T} P+H_{\mu} P+P H_{\mu}\right) & P \\
P & -V^{\mu} R^{-1}
\end{array}\right)
$$

is negative semidefinite. Indeed, multiplying the first inequality from (24) by $V^{-\mu}$ and applying the Schur complement, we obtain the LMI of the form

$$
\left(\begin{array}{cc}
V^{-\mu}\left(\tilde{A} X+X \tilde{A}^{T}+\tilde{B} Y+Y^{T} \tilde{B}^{T}+H_{\mu} X+X H_{\mu}\right) & I \\
I & -V^{\mu} R^{-1}
\end{array}\right) \leq 0
$$

which is equivalent to $W \leq 0$, since $X=P^{-1}$ and $K=Y X^{-1}$. The inequality (25) implies $\dot{V}=$ $-\left[\frac{\partial Q}{\partial V}\right]^{-1} \frac{\partial Q}{\partial s}(\tilde{A} s+\tilde{B} \tilde{u}+\tilde{\tilde{d}}) \leq\left[\frac{\partial Q}{\partial V}\right]^{-1} \frac{(1-\beta) s^{T} D_{r}\left(V^{-1}\right)\left(H_{\mu} P+P H_{\mu}\right) D_{r}\left(V^{-1}\right) s}{V^{\mu}}=-(1-\beta) V^{1-\mu}$.

The feasibility of the LMI (24) can be proven by analogy with Proposition 12.

The inequality (25), that restricts the system disturbances in the last theorem, has an implicit form, which is not appropriate for practice. Therefore, it is important to present such restriction to disturbance functions $\tilde{d}$ that can be easily analyzed.

Let the matrices $E_{i}, i=1,2, . . k$ be introduced by the formula

$$
E_{i}=\left(\begin{array}{ccccc}
0_{n_{1}} & \ldots & 0_{n_{1} \times n_{i}} & \ldots & 0_{n_{1} \times n_{k}} \\
\ldots & \ldots & \ldots & \ldots & \ldots \\
0_{n_{i} \times n_{1}} & \ldots & I_{n_{i}} & \ldots & 0_{n_{i} \times n_{k}} \\
\ldots & \ldots & \ldots & \ldots & \ldots \\
0_{n_{k} \times n_{1}} & \ldots & 0_{n_{k} \times n_{i}} & \ldots & 0_{n_{k}}
\end{array}\right) .
$$

It can be shown that if $E_{i} \tilde{d} \equiv 0$ for some $i=i_{1}, i_{2}, \ldots, i_{p}$ then Theorem 15 stays true even when the term $R$ in the LMI (16) is replaced with $E_{i_{p}} \ldots E_{i_{2}} E_{i_{1}} R E_{i_{1}} E_{i_{2}} \ldots E_{i_{p}}$. 
Proposition 16

Let $X \in \mathbb{R}^{n \times n}$ be a solution of the LMI system (24) with $R=I_{n}$ and $P=X^{-1}$. If

$$
\tilde{d}^{T} E_{i} \tilde{d} \leq \beta_{i} \gamma\left\{\begin{array}{lll}
\left(\lambda_{\min }(P) s^{T} s\right)^{1+(k-i-1) \mu} & \text { if } \quad s^{T} P s \leq 1, \\
\left(\lambda_{\min }(P) s^{T} s\right)^{\frac{1+(k-i-1) \mu}{1+(k-1) \mu}} & \text { if } \quad s^{T} P s>1,
\end{array}\right.
$$

for some $\beta_{i} \in \mathbb{R}_{+}: \beta=\beta_{1}+\ldots+\beta_{k}<1$ and $\gamma:=\lambda_{\min }\left(P^{1 / 2} H_{\mu} P^{-1 / 2}+P^{-1 / 2} H_{\mu} P^{1 / 2}\right)$ then the inequality (25) of Theorem 15 holds.

Proof

The definition of the number $\gamma$ implies $\gamma I_{n} \leq P^{1 / 2} H_{\mu} P^{-1 / 2}+P^{-1 / 2} H_{\mu} P^{1 / 2}$ or equivalently $\gamma P \leq P H_{\mu}+H_{\mu} P$. Hence, the definition of the implicit Lyapunov function gives

$$
\gamma=\gamma s^{T} D_{r}\left(V^{-1}\right) P D_{r}\left(V^{-1}\right) s \leq s^{T} D_{r}\left(V^{-1}\right)\left(P H_{\mu}+H_{\mu} P\right) D_{r}\left(V^{-1}\right) s,
$$

where $(V, s) \in \mathbb{R}_{+} \times \mathbb{R}^{n}$ such that $Q(V, s)=0$. On the other hand, we have

$$
1=s^{T} D_{r}\left(V^{-1}\right) P D_{r}\left(V^{-1}\right) s \geq\left\{\begin{array}{cl}
\lambda_{\min }(P) V^{-2} s^{T} s & \text { for } \quad s^{T} P s \leq 1 \\
\lambda_{\min }(P) V^{-2-2(k-1) \mu} s^{T} s & \text { for } \quad s^{T} P s>1 .
\end{array}\right.
$$

Hence, we derive $\tilde{d}^{T} D_{r}\left(V^{-1}\right) R^{-1} D_{r}\left(V^{-1}\right) \tilde{d}=\sum_{i=1}^{k} V^{-2-2(k-i) \mu} \tilde{d}^{T} E_{i} \tilde{d} \leq \frac{\gamma}{V^{2 \mu}}\left(\beta_{1}+\ldots+\beta_{k}\right) \leq$ $\beta V^{-2 \mu} s^{T} D_{r}\left(V^{-1}\right)\left(P H_{\mu}+H_{\mu} P\right) D_{r}\left(V^{-1}\right) s$, i.e. the inequality (25) holds.

Note that the condition (28) may be fulfilled only if the so-called unmatched disturbances are "vanishing" at the origin, i.e. $\tilde{d}^{T}(t, s) E_{i} \tilde{d}(t, s) \rightarrow 0$ as $s \rightarrow 0$ for $i=1,2, \ldots, k-1$. If $\mu=1$ then restriction to the so-called matched part of disturbances becomes $\tilde{d}^{T} E_{k} \tilde{d} \leq \beta_{k} \gamma$, i.e. the ILF control rejects bounded matched disturbances.

\subsection{Fixed-Time Stabilization}

In order to design fixed-time ILF control we consider two implicit Lyapunov functions defined by

$$
\begin{aligned}
& Q_{1}(V, s):=s^{T} D_{r_{\mu}}\left(V^{-1}\right) P D_{r_{\mu}}\left(V^{-1}\right) s-1, \\
& Q_{2}(V, s):=s^{T} D_{r_{\nu}}\left(V^{-1}\right) P D_{r_{\nu}}\left(V^{-1}\right) s-1,
\end{aligned}
$$

where $P \in \mathbb{R}^{n \times n}, P>0$ and $D_{r_{\mu}}(\lambda)=\left\{\lambda^{1+(k-i) \mu} I_{n_{i}}\right\}_{i=1}^{k}$ and $D_{r_{\nu}}(\lambda)=\left\{\lambda^{1+(i-1) \nu} I_{n_{i}}\right\}_{i=1}^{k}$ with $\lambda \in \mathbb{R}_{+}$. Denote $H_{\mu}=\operatorname{diag}\left\{(1+(k-i) \mu) I_{n_{i}}\right\}_{i=1}^{k}$ and $H_{\nu}=\operatorname{diag}\left\{(1+(i-1) \nu) I_{n_{i}}\right\}_{i=1}^{k}$.

\section{Theorem 17 (On fixed-time robust stabilization)}

If 1$)$ the system of linear matrix inequalities

$$
\begin{gathered}
\tilde{A} X+X \tilde{A}^{T}+\tilde{B} Y+Y^{T} \tilde{B}^{T}+\alpha_{\mu}\left(X H_{\mu}+H_{\mu} X\right)+R_{\mu} \leq 0, \\
\tilde{A} X+X \tilde{A}^{T}+\tilde{B} Y+Y^{T} \tilde{B}^{T}+\alpha_{\nu}\left(X H_{\nu}+H_{\nu} X\right)+R_{\nu} \leq 0, \\
X H_{\mu}+H_{\mu} X>0, \quad X H_{\nu}+H_{\nu} X>0, \quad X>0, \quad X \in \mathbb{R}^{n \times n}, \quad Y \in \mathbb{R}^{n_{k} \times n},
\end{gathered}
$$

is feasible for some fixed numbers $\mu \in(0,1], \nu, \alpha_{\mu}, \alpha_{\nu} \in \mathbb{R}_{+}$and some fixed matrices $R_{\nu}, R_{\mu} \in$ $\mathbb{R}^{n \times n}, R_{\mu}>0, R_{\nu}>0$

2) the control law $u$ has the form (11) with

$$
\tilde{u}=\tilde{u}(V, s)=\left\{\begin{array}{lll}
V^{1-\mu} K D_{r_{\mu}}\left(V^{-1}\right) s & \text { for } \quad s^{T} P s<1, \\
V^{1+k \nu} K D_{r_{\nu}}\left(V^{-1}\right) s & \text { for } \quad s^{T} P s \geq 1,
\end{array}\right.
$$

where $K=Y X^{-1}, P=X^{-1}$ and $V$ defined by

$$
V \in \mathbb{R}_{+}:\left\{\begin{array}{lll}
Q_{1}(V, s)=0 & \text { for } & s^{T} P s<1 \\
Q_{2}(V, s)=0 & \text { for } & s^{T} P s \geq 1
\end{array}\right.
$$


3) the disturbance function $\tilde{d}$ satisfies

$$
\begin{array}{ccc}
\tilde{d}^{T} D_{r_{\mu}}\left(V^{-1}\right) R_{\mu}^{-1} D_{r_{\mu}}\left(V^{-1}\right) \tilde{d} \leq \beta_{\mu} V^{-2 \mu} s^{T} D_{r_{\mu}}\left(H_{\mu} P+P H_{\mu}\right) D_{r_{\mu}} s & \text { if } \quad s^{T} P s \leq 1 \\
\tilde{d}^{T} D_{r_{\nu}}\left(V^{-1}\right) R_{\nu}^{-1} D_{r_{\nu}}\left(V^{-1}\right) \tilde{d} \leq \beta_{\nu} V^{2 \nu} s^{T} D_{r_{\nu}}\left(H_{\nu} P+P H_{\nu}\right) D_{r_{\nu}} s \quad \text { if } \quad s^{T} P s \geq 1
\end{array}
$$

for some $\beta_{\mu} \in\left[0, \alpha_{\mu}\right)$ and $\beta_{\nu} \in\left[0, \alpha_{\nu}\right)$, then the closed-loop system (1) is globally fixed-time stable with the following estimate of the settling time function:

$$
T\left(x_{0}\right) \leq \frac{1}{\left(\alpha_{\mu}-\beta_{\mu}\right) \mu}+\frac{1}{\left(\alpha_{\nu}-\beta_{\nu}\right) \nu} .
$$

Proof

Following the proof of Theorem 10 we can show that $Q_{1}(V, s)$ and $Q_{2}(V, s)$ satisfy the conditions C1)-C4) of Theorem 7. Obviously, $Q_{1}(1, s)=Q_{2}(1, s)$ and the condition C6) of Theorem 8 holds. In this case the formula (32) implicitly defines the Lyapunov function candidate $V: \mathbb{R}^{n} \rightarrow \mathbb{R}_{+}$, which can be prolonged by continuity to the origin $V(0)=0$. Remark that $s^{T} P s \leq 1 \Rightarrow V(s) \leq 1$ and $s^{T} P s \geq 1 \Rightarrow V(s) \geq 1$.

Similarly to the proof of Theorem 10 it can be shown

$$
-\left[\frac{\partial Q_{1}}{\partial V}\right]^{-1} \frac{\partial Q_{1}}{\partial s}(\tilde{A} s+\tilde{B} \tilde{u}+\tilde{d}) \leq-\left(\alpha_{\mu}-\beta_{\mu}\right) V^{1-\mu} \quad \text { for } \quad V(s) \leq 1 .
$$

For the function $Q_{2}(V, s)$ we have $\frac{\partial Q_{2}}{\partial V}=-V^{-1} s^{T} D_{r_{\nu}}\left(V^{-1}\right)\left(H_{\nu} P+P H_{\nu}\right) D_{r_{\nu}}\left(V^{-1}\right) s$ and $\frac{\partial Q_{2}}{\partial s}(\tilde{A} s+\tilde{B} \tilde{u}+\tilde{d})=2 s^{T} D_{r_{\nu}}\left(V^{-1}\right) P D_{r_{\nu}}\left(V^{-1}\right)(\tilde{A} s+\tilde{B} \tilde{u}+\tilde{d})$. Taking into account that $D_{r_{\nu}}\left(V^{-1}\right) \tilde{A} D_{r_{\nu}}^{-1}\left(V^{-1}\right)=V^{\nu} \tilde{A}$ and $D_{r_{\nu}}\left(V^{-1}\right) \tilde{B} \tilde{u}=V^{\nu} \tilde{B} K D_{r_{\nu}}\left(V^{-1}\right) s$ for $s^{T} P s \geq 1$ we obtain

$$
\begin{gathered}
\frac{\partial Q_{2}}{\partial s}(\tilde{A} s+\tilde{B} \tilde{u}+\tilde{d})=V^{\nu} s^{T} D_{r_{\nu}}\left(V^{-1}\right)\left(P(\tilde{A}+\tilde{B} K)+(\tilde{A}+\tilde{B} K)^{T} P\right) D_{r_{\nu}}\left(V^{-1}\right) s+ \\
2 s^{T} D_{r_{\nu}}\left(V^{-1}\right) P D_{r_{\nu}}\left(V^{-1}\right) \tilde{d}=\left(\begin{array}{c}
D_{r_{\nu}}\left(V^{-1}\right) s \\
D_{r_{\nu}}\left(V^{-1}\right) \tilde{d}
\end{array}\right)^{T} W_{2}\left(\begin{array}{c}
D_{r_{\nu}}\left(V^{-1}\right) x \\
D_{r_{\nu}}\left(V^{-1}\right) \tilde{d}
\end{array}\right)+ \\
\alpha_{\nu} V^{\nu} s^{T} D_{r_{\nu}}\left(V^{-1}\right)\left(H_{\nu} P+P H_{\nu}\right) D_{r_{\nu}}\left(V^{-1}\right) s+V^{-\nu} \tilde{d}^{T} D_{r_{\nu}}\left(V^{-1}\right) R_{\nu}^{-1} D_{r_{\nu}}\left(V^{-1}\right) \tilde{d}
\end{gathered}
$$

where

$$
W_{2}=\left(\begin{array}{cc}
V^{\nu}\left(P(\tilde{A}+\tilde{B} K)+(\tilde{A}+\tilde{B} K)^{T} P+\alpha_{\nu}\left[H_{\nu} P+P H_{\nu}\right]\right) & P \\
P & -V^{-\nu} R_{\nu}^{-1}
\end{array}\right) \leq 0 .
$$

Hence

$$
\dot{V}=-\left[\frac{\partial Q_{2}}{\partial V}\right]^{-1} \frac{\partial Q_{2}}{\partial s}(\tilde{A} S+\tilde{B} \tilde{u}+\tilde{d}) \leq-\left(\alpha_{\nu}-\beta_{\nu}\right) V^{1+\nu} \quad \text { for } \quad V(s) \geq 1
$$

Therefore, all conditions of Theorem 8 hold.

The parameters $\alpha_{\mu}$ and $\alpha_{\nu}$ are introduced to the LMI system (30) for tuning of the convergence time of the closed-loop system.

Similarly to the finite-time case it is easy to check that the disturbance-free system (12), (31) is homogeneous in the locally homogeneous with negative degree $-\mu$ at 0 -limit and locally homogeneous with positive degree $\nu$ in $\infty$-limit. The Input-to-State Stability analysis of the systems that is homogeneous in the bi-limit can be found in [9], [21]. The feasibility of the LMI (30) can be proven analogously to Proposition 12.

Proposition 18

Let $X \in \mathbb{R}^{n \times n}$ be a solution of the LMI system (30) with $R_{\mu}=R_{\nu}=I_{n}$ and $P=X^{-1}$. If for $i=1,2, \ldots, k$

$$
\tilde{d}^{T} E_{i} \tilde{d} \leq\left\{\begin{array}{cc}
\beta_{\mu}^{i} \gamma_{\mu}\left(\lambda_{\min }(P) s^{T} s\right)^{1+(k-i-1) \mu} & \text { if } \quad s^{T} P s \leq 1, \\
\beta_{\nu}^{i} \gamma_{\nu}\left(\lambda_{\min }(P) s^{T} s\right)^{\frac{1+i \nu}{1+(k-1) \nu}} & \text { if } \quad s^{T} P s>1,
\end{array} \quad i=1,2, \ldots, k,\right.
$$


where $\beta_{\mu}^{i} \in \mathbb{R}_{+}$and $\beta_{\nu}^{i} \in \mathbb{R}_{+}, \beta_{\mu}=\beta_{\mu}^{1}+\ldots+\beta_{\mu}^{k}<\alpha_{\mu}$ and $\beta_{\nu}=\beta_{\nu}^{1}+\ldots+\beta_{\nu}^{k}<\alpha_{\nu}, \gamma_{\mu}=$ $\lambda_{\min }\left(P^{1 / 2} H_{\mu} P^{-1 / 2}+P^{-1 / 2} H_{\mu} P^{1 / 2}\right)$ and $\gamma_{\nu}=\lambda_{\min }\left(P^{1 / 2} H_{\nu} P^{-1 / 2}+P^{-1 / 2} H_{\nu} P^{1 / 2}\right)$, then the condition 3 ) of Theorem 17 holds.

Proof

The case $s^{T} P s \leq 1$ can be studied similarly to the proof of proposition 16 . Consider the case $s^{T} P s>1$ in this case the implicit Lyapunov function $V=V(s)$ is defined by the equation $Q_{2}(V, s)=0$ and $1=s^{T} D_{r_{\nu}}\left(V^{-1}\right) P D_{r_{\nu}}\left(V^{-1}\right) s \geq \lambda_{\min }(P) V^{-2-2(k-1) \nu} s^{T} s$. For $R_{\nu}=I$ we have $\tilde{d}^{T} D_{r_{\nu}}\left(V^{-1}\right) R_{\nu}^{-1} D_{r_{\nu}}\left(V^{-1}\right) \tilde{d}=\sum_{i=1}^{k} \frac{\tilde{d}^{T} E_{i} \tilde{d}}{V^{2+2(i-1) \nu}} \leq \beta_{\nu} V^{2 \nu} s^{T} D_{r_{\nu}}\left(V^{-1}\right)\left(H_{\nu} P+P H_{\mu}\right) D_{r_{\nu}}\left(V^{-1}\right) s$.

The fixed-time ILF control algorithm may reject a wider class of disturbances comparing with the finite-time one. For example, the linear disturbance function $\tilde{d}=\Delta s, \Delta \in \mathbb{R}^{n \times n}$ never satisfies the condition (28), but, obviously, the condition (35) will be fulfilled for sufficiently small $\|\Delta\|$ and $k \leq 2$. It is also worth to stress that the conditions (25), (28), (33), (35) can be considered locally if the operation domain is known a-priori.

\section{Remark 19}

The theorems 15 and 17 have been proven for continuous disturbance function $\tilde{d}$. They can be easily extended to the class of piecewise continuous (with respect to state variable) functions. In this case, the conditions of the theorems must hold for any selector $\tilde{d}^{\prime} \in \tilde{D}$, where $\tilde{D}$ is the set-valued mapping defined by Filippov regularization procedure as follows

$$
\tilde{D}(t, s)=\bigcap_{\delta>0} \bigcap_{N: \mu(N)=0} \operatorname{co} \tilde{d}(t, s+B(\delta) \backslash N),
$$

where $\operatorname{co}(N)$ defines the convex closure of the set $N \subset \mathbb{R}^{n}$ and the equality $\mu(N)=0$ means that the set $N$ has measure 0 .

\section{ON PRACTICAL IMPLEMENTATION OF THE CONTROL ALGORITHM}

\subsection{Sampled-Time ILF Control}

In order to realize the control algorithm (17) in practice we need to know $V(s)$. In some cases the function $V(s)$ can be calculated analytically, for example, for $n=2, m=1$. However, even for the second order case this representation is very cumbersome. The function $V(s)$ can also be approximated numerically on a grid, which is constructed in some operation domain (a neighborhood of the origin). Moreover, the ILF control can be easily implemented to linear control systems that admit the on-line variation of the feedback gains. Indeed, for any fixed $V_{0}$ the control $\tilde{u}\left(V_{0}, s\right)$ defined by (17) or (31) becomes linear feedback. Denote

$$
\Pi_{\mu}(V, P):=\left\{z \in \mathbb{R}^{n}: z^{T} D_{\mu}\left(V^{-1}\right) P D_{\mu}\left(V^{-1}\right) z \leq 1\right\} .
$$

Theorem 20

Let the conditions of Theorem 15 hold and the disturbance function $\tilde{d}$ satisfies the condition (25). If the control $\tilde{u}=\tilde{u}\left(V_{0}, s\right)$ is defined by (17) with an arbitrary fixed positive number $V_{0} \in \mathbb{R}_{+}$ then the ellipsoid $\Pi_{\mu}\left(V_{0}, X^{-1}\right)$ is positively invariant set of the closed-loop system (1), (11), i.e $s\left(t^{\prime}\right) \in \Pi_{\mu}\left(V_{0}, X^{-1}\right) \quad \Rightarrow \quad s(t) \in \Pi_{\mu}\left(V_{0}, X^{-1}\right)$ for all $t>t^{\prime}$.

Proof

I. Rewrite the matrix inequality (24) with $X=P^{-1}$ and $k=y X^{-1}$ in the form:

$$
\left(\tilde{A}+H_{\mu}\right)^{T} P+P\left(\tilde{A}+H_{\mu}\right)+P \tilde{B} K+K^{T} \tilde{B}^{T} P+P R P \leq 0 .
$$

Hence, we have

$$
D_{r}\left(V_{0}^{-1}\right)\left(\left(\tilde{A}+H_{\mu}\right)^{T} P+P\left(\tilde{A}+H_{\mu}\right)+P \tilde{B} K+K^{T} \tilde{B}^{T} P+P R P\right) D_{r}\left(V_{0}^{-1}\right) \leq 0 .
$$


Denoting $P_{0}:=D_{r}\left(V_{0}^{-1}\right) P D_{r}\left(V_{0}^{-1}\right)>0$ and taking into account $D_{r}^{-1}\left(V_{0}^{-1}\right) \tilde{A} D_{r}\left(V_{0}^{-1}\right)=V_{0}^{\mu} \tilde{A}$, $D_{r}^{-1}\left(V_{0}^{-1}\right) H_{\mu} D_{r}\left(V_{0}^{-1}\right)=H_{\mu}$ and $D_{r}^{-1}\left(V_{0}^{-1}\right) \tilde{B}=V_{0} \tilde{B}$ we derive

$$
P_{0} \tilde{A}+\tilde{A}^{T} P_{0}+P_{0} \tilde{B} K_{0}+K_{0}^{T} \tilde{B}^{T} P_{0}+\frac{1}{V_{0}^{\mu}}\left(H_{\mu} P_{0}+P_{0} H_{\mu}+P_{0} D_{\mu}\left(V_{0}\right) R D_{\mu}\left(V_{0}\right) P_{0}\right) \leq 0,
$$

where $K_{0}=V_{0}^{1-\mu} k D_{r}\left(V_{0}^{-1}\right)$.

II. Consider the following Lyapunov function candidate $\tilde{V}(s)=s^{T} P_{0} s$. Since $\tilde{u}_{0}(s)=\tilde{u}\left(V_{0}, s\right)=$ $K_{0} s$ then

$$
\left.\frac{d \tilde{V}}{d t}\right|_{(1)}=\left(\begin{array}{c}
s \\
\tilde{d}
\end{array}\right)^{T} W_{0}\left(\begin{array}{c}
s \\
\tilde{d}
\end{array}\right)-V_{0}^{-\mu} s^{T}\left(P_{0} H_{\mu}+H_{\mu} P_{0}\right) s+V_{0}^{\mu} \tilde{d}^{T} D_{\mu}\left(V_{0}^{-1}\right) R^{-1} D_{\mu}\left(V_{0}^{-1}\right) \tilde{d}
$$

where

$$
W_{0}=\left(\begin{array}{cc}
P_{0} \tilde{A}+\tilde{A}^{T} P_{0}+P_{0} \tilde{B} K_{0}+K_{0}^{T} \tilde{B}^{T} P_{0}+\frac{1}{V_{0}^{\mu}}\left(H_{\mu} P_{0}+P_{0} H_{\mu}\right) & P_{0} \\
P_{0} & -V_{0}^{\mu}\left[D_{\mu}\left(V_{0}\right) R D_{\mu}\left(V_{0}\right)\right]^{-1}
\end{array}\right) .
$$

The Schur complement implies $W_{0} \leq 0$ and taking into account the condition (25) we obtain

$$
\left.\frac{d \tilde{V}}{d t}\right|_{(12)} \leq-\frac{1-\beta}{V_{0}^{\mu}} s^{T}\left(P_{0} H_{\mu}+H_{\mu} P_{0}\right) s \quad \forall s \in \mathbb{R}^{n}: Q\left(V_{0}, s\right)=0\left(\text { i.e. } s^{T} P_{0} s=1\right),
$$

where $\beta \in(0,1)$. Therefore, the ellipsoid $\Pi_{\mu}\left(V_{0}, P\right)$ is strictly positively invariant set of the closedloop system (1) with the control $\tilde{u}:=\tilde{u}\left(V_{0}, s\right)$.

Now we assume that the value $V$ can be changed only in some sampled time instances and let us show the robustness of the sampled-time implementation of the ILF control algorithm.

\section{Corollary 21 (On sampled-time ILF control realization)}

If

1) the conditions of Theorem 15 hold and the disturbance function $\tilde{d}$ satisfies the condition (25);

2) $\left\{t_{i}\right\}_{i=0}^{+\infty}$ is an arbitrary sequence of time instances such that

$$
0=t_{0}<t_{1}<t_{2}<\ldots \quad \text { and } \quad \lim _{i \rightarrow+\infty} t_{i}=+\infty
$$

3) the control has the form (11) with $\tilde{u}=\tilde{u}\left(V_{i}, s\right)$ on each time interval $\left[t_{i}, t_{i+1}\right)$, where $\tilde{u}(V, s)$ is defined by (17) and $V_{i} \in R_{+}: Q\left(V_{i}, s\left(t_{i}\right)\right)=0$;

then the closed-loop system (1) is globally asymptotically stable.

Proof

Let $V(s)$ be a positive definite function implicitly defined by the equation $Q(V, s)=0$. In this case we have $V_{i}=V\left(s\left(t_{i}\right)\right)$.

I. Let us prove that the sequence $\left\{V_{i}\right\}_{i=1}^{+\infty}$ is monotone decreasing. Consider the time interval $t \in\left[t_{i}, t_{i+1}\right)$ and the quadratic function $\tilde{V}_{i}(s):=s^{T} P_{i} s$, where $P_{i}:=D_{r}\left(V_{i}^{-1}\right) P D_{r}\left(V_{i}^{-1}\right)>0$ and $P=X^{-1}$ defined by (24).

The switching control $\tilde{u}(s)$ on this interval takes the form $\tilde{u}_{i}(s)=\tilde{u}\left(V_{i}, s\right)=K_{i} s$, where $K_{i}:=$ $V_{i}^{1-\mu} K D_{r}\left(V_{i}^{-1}\right)$. Repeating the proof of Theorem 20 we derive $\left.\frac{d \tilde{V}_{i}}{d t}\right|_{(12)} \leq-\frac{1-\beta}{V_{i}^{\mu}} s^{T}\left(P_{i} H_{\mu}+\right.$ $\left.H_{\mu} P_{i}\right) s$ for $s \in \mathbb{R}^{n}: Q\left(V_{i}, s\right)=0$ and $t \in\left[t_{i}, t_{i+1}\right)$, where $\beta \in(0,1)$. Hence, $\left.\frac{d \tilde{V}_{i}\left(s\left(t_{i}\right)\right)}{d t}\right|_{(12)}<0$ and $\tilde{V}_{i}(s(t))<\tilde{V}_{i}\left(s\left(t_{i}\right)\right)$ for all $\left(t_{i}, t_{i+1}\right]$. In this case we have

$$
\begin{gathered}
Q\left(V_{i}, s(t)\right)=s^{T}(t) D_{r}\left(V_{i}^{-1}\right) P D_{r}\left(V_{i}^{-1}\right) s(t)-1= \\
\tilde{V}_{i}(s(t))-1<\tilde{V}_{i}\left(s\left(t_{i}\right)\right)-1=Q\left(V_{i}, s\left(t_{i}\right)\right)= \\
0=Q(V(s(t)), s(t)), \forall t \in\left(t_{i}, t_{i+1}\right] .
\end{gathered}
$$


For any given $s \in \mathbb{R}^{n} \backslash\{0\}$ the function $Q(V, s)$ is monotone decreasing for all $V \in \mathbb{R}^{+}$(see Condition C4) of Theorem 7). Then the obtained inequality implies $V(s(t))<V\left(s\left(t_{i}\right)\right), \forall t \in$ $\left(t_{i}, t_{i+1}\right]$, i.e. the sequence $\left\{V_{i}\right\}_{i=1}^{+\infty}$ is monotone decreasing and $s(t) \in \Pi_{\mu}\left(V_{i}, P\right)$ for $t \geq t_{i}$. Moreover, $V(s(t)) \leq V(s(0))$ for all $t \geq 0$, i.e. the origin of the system (1) is Lyapunov stable.

II. Since the function $V(s)$ is positive definite then the monotone decreasing sequence $\left\{V\left(s\left(t_{i}\right)\right)\right\}_{i=1}^{\infty}$ converge to some limit. Let us show now that this limit is zero. Suppose the contrary, i.e. $\lim _{i \rightarrow \infty} V\left(s\left(t_{i}\right)\right)=V_{*}>0$ or equivalently $\forall \varepsilon>0 \quad \exists N=N(\varepsilon): V_{*} \leq V_{i}<V_{*}+\varepsilon, \forall i \geq N$.

The control function $u(V, s)$ is continuous $\forall s \in \mathbb{R}^{n} \backslash\{0\}$ and $\forall V \in \mathbb{R}_{+}$. Then we have

$$
\left\|u\left(V_{i}, s\right)-u\left(V_{*}, s\right)\right\|=\left\|V_{i}^{1-\mu} k D_{r}\left(V_{i}^{-1}\right) s-\left(V_{*}\right)^{1-\mu} k D_{r}\left(V_{*}^{-1}\right) s\right\| \leq \sigma(\varepsilon)\|s\|, \quad \forall i \geq N,
$$

where $\sigma(\cdot) \in \mathcal{K}$. This means that for $t>t_{N}$ the closed-loop system (1) can be presented in the form

$$
\dot{s}=\tilde{A} s+\tilde{B}\left(K_{*}+\Delta(t, \varepsilon)\right) s+\tilde{d},
$$

where $K_{*}=V_{*}^{1-\mu} K D_{r}\left(V_{*}^{-1}\right)$ and $\Delta(t, \varepsilon) \in \mathbb{R}^{m \times n}:\|\Delta\| \leq \sigma(\varepsilon)$.

Consider the quadratic Lyapunov function candidate $\tilde{V}_{*}(s)=s^{T} P_{*} s$, where $P_{*}=$ $D_{r}\left(V_{*}^{-1}\right) P D_{r}\left(V_{*}^{-1}\right)$. For $t>t_{N}$ we have

$$
\left.\frac{d \tilde{V}_{*}}{d t}\right|_{(12)} \leq-V_{*}^{-\mu} s^{T}\left(P_{*} H_{\mu}+H_{\mu} P_{*}\right) s+s^{T}\left(P_{*} \tilde{B} \Delta+\Delta^{T} \tilde{B}^{T} P_{*}\right) s+V_{*}^{\mu} \tilde{d}^{T} D_{\mu}\left(V_{*}^{-1}\right) R D_{\mu}\left(V_{*}^{-1}\right) \tilde{d} .
$$

Introduce the set

$$
\Omega(\varepsilon)=\bigcup_{\delta \in(-\varepsilon, \varepsilon)}\left\{z \in \mathbb{R}^{n}: z^{T} D_{\mu}\left(\left(V_{*}+\delta\right)^{-1}\right) P D_{\mu}\left(\left(V_{*}+\delta\right)^{-1}\right) z=1\right\} .
$$

The condition (25) implies that for sufficiently small $\varepsilon \in \mathbb{R}_{+}$and $\forall s \in \Omega(\varepsilon)$ we have

$$
V_{*}^{\mu} \tilde{d}^{T} D_{\mu}\left(V_{*}^{-1}\right) R D_{\mu}\left(V_{*}^{-1}\right) \tilde{d} \leq \beta_{*} V_{*}^{-\mu} s^{T}\left(H_{\mu} P_{*}+P_{*} H_{\mu}\right) s,
$$

where $0 \leq \beta \leq \beta_{*}(\varepsilon)<1$.

Hence, for $t>t_{N}$ we derive

$$
\left.\frac{d \tilde{V}_{*}}{d t}\right|_{(12)} \leq-\frac{1-\beta_{*}(\varepsilon)}{V_{*}^{\mu}} s^{T}\left(P_{*} H_{\mu}+H_{\mu} P_{*}\right) s+s^{T}\left(P_{*} \tilde{B} \Delta+\Delta^{T} \tilde{B}^{T} P_{*}\right) s \leq-\left(\frac{1-\beta_{*}(\varepsilon)}{V_{*}^{\mu}} \gamma_{*}-\gamma(\varepsilon)\right) \tilde{V}^{*},
$$

where $\gamma_{*}=\lambda_{\min }\left(P_{*}^{1 / 2} H_{\mu} P_{*}^{-1 / 2}+P_{*}^{-1 / 2} H_{\mu} P_{*}^{1 / 2}\right), \gamma(\varepsilon)=\lambda_{\max }\left(P_{*}^{1 / 2} \tilde{B} \Delta P_{*}^{-1 / 2}+P_{*}^{-1 / 2} \Delta^{T} \tilde{B}^{T} P_{*}^{1 / 2}\right)$ and $\gamma(\varepsilon) \in \mathcal{K}$. Therefore, for sufficiently small $\varepsilon$ the Lyapunov function $\tilde{V}_{*}(t)$ is exponentially decreasing for all $t>t_{N}$ and there exists $t^{*} \geq t_{N}$ such that $\tilde{V}_{*}\left(t_{*}\right)=V_{*}$ and $\tilde{V}_{*}(t)<V_{*}$ for $t>t_{*}$. This contradicts with our assumption and means $\lim _{i \rightarrow \infty} V_{i}=0$ implying that the closedloop system (1) with sampled-time implementation of the ILF control algorithm is globally asymptotically stabile.

The proven corollary guarantees that the sampled-time ILF control provides robust asymptotic stabilization of the closed-loop system (1) independently on the length of the sampling interval. Such property is rather unusual for sampled and switched control systems with additive disturbances.

Theorem 20 and Corollary 21 can be easily extended to the case of fixed-time ILF control application.

\subsection{Digital Implementation}

The ILF control implementation requires solving the equation $Q(V, s)=0$ numerically and on-line in order to find an appropriate value of $V_{i}$ at the time instant $t_{i}$. Fortunately, for practical reasons rather simple numerical procedures can be utilized.

Denote as before $V_{i}:=V\left(t_{i}\right)$ and $s_{i}:=s\left(t_{i}\right)$ and suppose that the control $\tilde{u}$ has the form $\tilde{u}\left(V_{i}, s\right)$ on the time interval $\left[t_{i}, t_{i+1}\right)$, where $0=t_{0}<t_{1}<t_{2}<\ldots$ and $\lim t_{i}=+\infty$.

The scheme for selection of the switching parameter $V_{i}$ is presented by the next algorithm [17]. 
Algorithm 22 ([17])

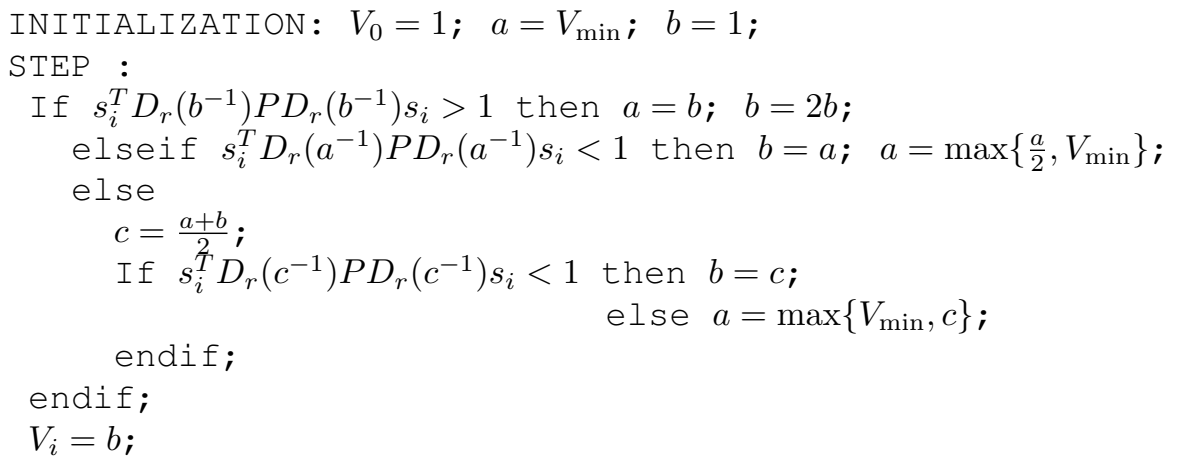

If $s_{i} \in \mathbb{R}^{n}$ is some given vector and STEP of the presented algorithm is applied recurrently many times to the same $s_{i}$ then Algorithm 22 realizes:

1) a localization of the unique positive root of the equation $Q\left(V, s_{i}\right)=0$, i.e. $V_{i} \in[a, b]$;

2) improvement of the obtained localization by means of the bisection method, i.e. $(b-a) \rightarrow 0$.

Such an application of Algorithm 22 allows us to calculate $V_{i}$ with rather high precision but it requests a high computational capability of a control device. If the computational power is very restricted, then STEP of Algorithm 22 may be realized just once at each sampled instant of time. Theorem 20 implies practical stability of the closed-loop system (1), (11) with sampled-time realization of ILF control and on-line calculation of the parameter $V_{i}$ by Algorithm 22. Indeed, Theorem 20 proves that $\Pi_{\mu}\left(V_{i}, s\right)$ is an invariant set of the closed-loop system (1), (11) with $\tilde{u}=\tilde{u}\left(V_{i}, P\right)$. When the root of the equation $Q\left(V, s_{i}\right)=0$ is localized, Algorithm 22 always selects the upper estimate of $V_{i}$ providing that $s\left(t_{i}\right) \in \Pi_{\mu}\left(V_{i}, s\right)$, i.e. $V_{i}$ do not increase in time even when $s_{i}=s\left(t_{i}\right)$ varies in time.

The parameter $V_{\min }$ defines lower admissible value of $V$. In practice, this parameter cannot be selected arbitrary small due to finite numerical precision of digital devices and measurement errors, which may imply $s\left(t_{i}\right) \notin \Pi_{\mu}\left(V_{i}, P\right)$. Therefore, the real-life realization of the ILF control provides the practical stabilization of the system with the attractive set $\Pi_{\mu}\left(V_{\min }, P\right)$.

The sampled-time realization of ILF control transforms the originally nonlinear closed-loop system to a switched linear system. This essentially simplifies the stability analysis of discretized fixed-time control algorithms.

A more detailed elaboration of sampled-time and discrete-time ILF control algorithms goes out of the scope of this paper. It can be done, for example, using the ideas of the paper [34].

\section{EXAMPLE: STABILIZATION OF DOUBLE INVERTED PENDULUM}

\subsection{Model Description}

Consider the mechanical system consisting of two connected inverted pendulums as it is shown on Figure 1. The dynamics of this mechanical system is described by the equation [35]

$$
H(q) \ddot{q}+F(q, \dot{q})=u+d(t),
$$

where $q=\left(q_{1}, q_{2}\right)^{T} \in \mathbb{R}^{n}$ - inclination angles of the links, $u \in \mathbb{R}^{2}$ is the vector of control inputs, the function $d \in \mathbb{R}_{+} \rightarrow \mathbb{R}^{2}$ describes the bounded exogenous disturbances,

$$
H=\left[\begin{array}{cc}
J_{1}+I_{1}+m_{1} l_{1}^{2}+m_{2} L_{1}^{2} & m_{2} L_{1} l_{2} \cos \left(q_{1}-q_{2}\right) \\
m_{2} L_{1} l_{2} \cos \left(q_{1}-q_{2}\right) & m_{2} l_{2}^{2}+I_{2}+J_{2}
\end{array}\right], F=\left[\begin{array}{c}
M \dot{q}_{2}^{2}-g\left(m_{1} l_{1}+m_{2} L_{1}\right) \sin q_{1} \\
-M \dot{q}_{1}^{2}-m_{2} g l_{2} \sin q_{2}
\end{array}\right],
$$

$M=m_{2} L_{1} l_{2} \sin \left(q_{1}-q_{2}\right), J_{i}$ is inertia of the rotor of the electric drive controlling $i$-th link, $m_{i}, I_{i}$ are mass and inertia of each link, $L_{i}$ is the total length of link and $l_{i}$ is the distance from the center mass of each link to its pivot point, $g=9.8$. 


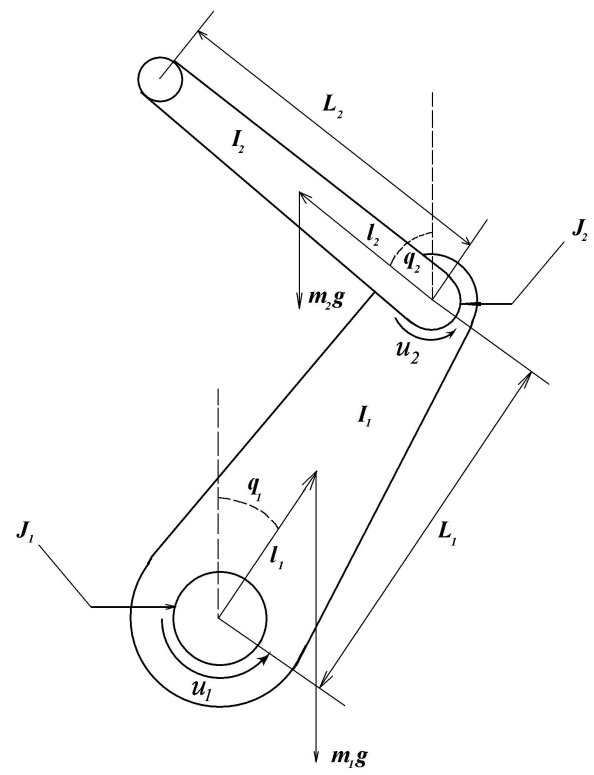

Figure 1: Double inverted pendulum

The inertia matrix $H(q)$ is always positive definite and consequently invertible. Denoting $x=$ $\left(x_{1}, x_{2}, x_{3}, x_{4}\right)^{T}=\left(q_{1}, q_{2}, \dot{q}_{1}, \dot{q}_{2}\right)^{T} \in \mathbb{R}^{4}$ let us rewrite the system (38) in the form

$$
\dot{x}=\left(\begin{array}{cc}
0 & I_{2} \\
0 & 0
\end{array}\right) x+\left(\begin{array}{c}
0 \\
S
\end{array}\right) \tilde{u}+\tilde{d},
$$

where $\tilde{d}=\left[H^{-1}\left(x_{1}, x_{2}\right)-S\right] u+H^{-1}\left(x_{1}, x_{2}\right)(d-F(x)), S=\operatorname{diag}\left\{\frac{1}{J_{1}+I_{1}+m_{1} l_{1}^{2}+m_{2} L_{1}^{2}}, \frac{1}{J_{2}+I_{2}+m_{2} l_{2}^{2}}\right\}$. In order to demonstrate the robustness of the ILF control we will consider $\tilde{d}$ as an unknown function.

\subsection{Simulation Results}

The following parameters of the double inverted pendulum have been used for simulations:

$$
\begin{gathered}
m_{1}=0.132, m_{2}=0.088, L_{1}=0.3032, L_{2}=0.3545, l_{1}=0.1274, \\
l_{2}=0.1209, I_{1}=0.0562, I_{2}=0.0314, J_{1}=6 \cdot 10^{-6}, J_{2}=3 \cdot 10^{-6} .
\end{gathered}
$$

Solving the system of matrix inequalities (24) for $\mu=1$ and $R=\operatorname{diag}\{0,0,1,1\}$ together with (23) for $u_{0}=5$ we obtain

$$
P=\left(\begin{array}{llll}
0.0099 & 0.0000 & 0.0043 & 0.0000 \\
0.0000 & 0.0024 & 0.0000 & 0.0010 \\
0.0043 & 0.0000 & 0.0022 & 0.0000 \\
0.0000 & 0.0010 & 0.0000 & 0.0005
\end{array}\right), K=-\left(\begin{array}{llll}
0.3064 & 0.0000 & 0.1994 & 0.0000 \\
0.0000 & 0.1507 & 0.0000 & 0.0981
\end{array}\right)
$$

We suppose that the magnitudes of control inputs are bounded: $\left|u_{1}\right| \leq 3$ and $\left|u_{2}\right| \leq 2$. The exogenous disturbances are selected as follows $d(t)=0.5[\sin (6 t), \cos (6 t)]^{T}$. For simulations it was assumed that the system states can be measured with a noise. The measurement noise was generated as a sequence of pseudorandom values drawn from the uniform distribution on the open interval $(-\delta, \delta)$. We select $\delta=0.001$ for the noised measurements of the angles and $\delta=0.01$ for angular velocities. Algorithm 22 has been used for ILF control application with $V_{\min }=0.05$ and $t_{i}=0.1 i$ 
for $i=0,1,2, \ldots$. Numerical simulations have been done using explicit Euler method with the step $h=10^{-3}$ and the following initial conditions $q_{1}(0)=\pi, q_{2}(0)=-\pi / 2, \dot{q}_{1}(0)=\dot{q}_{2}(0)=0, V_{0}=1$.

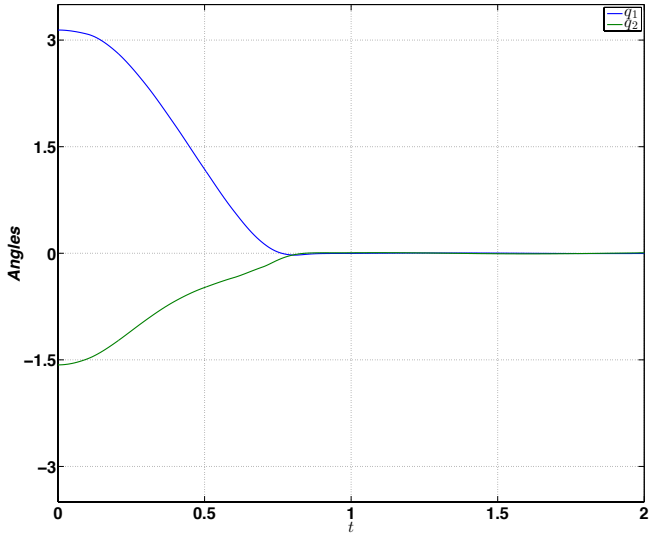

(a) Angular positions of the links.

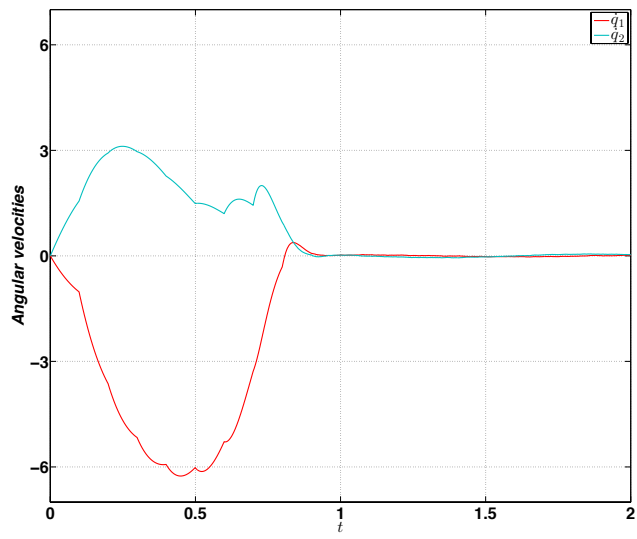

(b) Angular velocities of the links.

Figure 2: Evolution of the system states

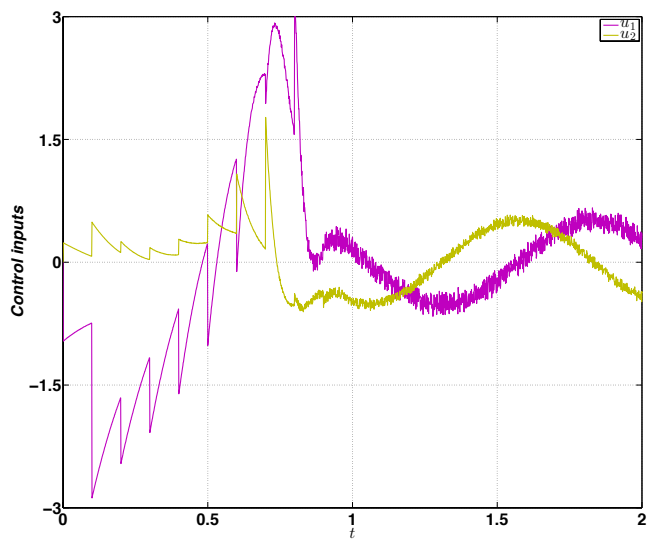

(a) Control torques.

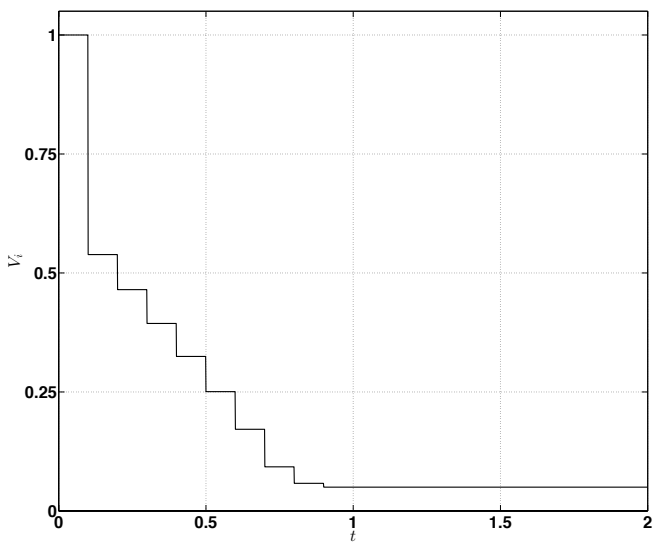

(b) Value of the sampled ILF.

Figure 3: Evolution of the control

Figures 2(a) and 2(b) show the evolution of the angles and angular velocities of the links for the ILF controlled double inverted pendulum. The control inputs are depicted on Figure 3(a). The sampled values of implicit Lyapunov function calculated by Algorithm 22 are presented on Figure 3(b). The simulation results approve good performances of the ILF control.

The settling-time function (18) obtained for the disturbance-free case provides $T\left(x_{0}\right)=0.5675$. The sampled-time realization of ILF control definitely brakes the finite-time convergence property to the origin, so the theoretical settling-time can be compared only with an estimate of the convergence time to some zone. Let us use for this comparison the convergence time of the ILF value to its minimal value $V_{\min }$. For the sampling period considered above the corresponding time is 0.9 . In order to obtain more precise estimate of the settling-time, the simulations of the disturbed system have been also done for the smaller sampling period 0.001 provided the estimate 0.671 .

\section{CONCLUSION}

The paper presents control algorithms for robust stabilization of linear plants provided nonasymptotic transitions. The control design procedures utilize the ILF method. This approach allows 
us to design the control together with the Lyapunov function and to provide constructive procedures for tuning of control parameters, which are presented in the form of LMIs. The scheme of the sampled-time ILF control implementation is developed. The robustness of the presented scheme is proven for arbitrary sampling period. The effectiveness of the ILF control for stabilization of disturbed double inverted pendulum is demonstrated on numerical simulations.

\section{REFERENCES}

1. E. Roxin, "On finite stability in control systems," Rendiconti del Circolo Matematico di Palermo, vol. 15(3), pp. 273-283, 1966.

2. V. Haimo, "Finite time controllers," SIAM Journal of Control and Optimization, vol. 24(4), pp. 760-770, 1986.

3. Y. Hong, "Finite-time stabilization and stabilizability of a class of controllable systems," Systems \& Control Letters, vol. 46, no. 4, pp. 231-236, 2002.

4. S. Bhat and D. Bernstein, "Finite-time stability of continuous autonomous systems," SIAM Journal of Control and Optimization, vol. 38(3), pp. 751-766, 2000.

5. E. Moulay and W. Perruquetti, "Finite-time stability and stabilization: State of the art," Lecture Notes in Control and Information Sciences, vol. 334, pp. 23-41, 2006.

6. A. Polyakov, "Nonlinear feedback design for fixed-time stabilization of linear control systems," IEEE Transactions on Automatic Control, vol. 57(8), pp. 2106-2110, 2012.

7. E. Moulay and W. Perruquetti, "Finite time stability and stabilization of a class of continuous systems," Journal of Mathematical Analysis and Application, vol. 323, no. 2, pp. 1430-1443, 2006.

8. A. Levant, "Homogeneity approach to high-order sliding mode design," Automatica, vol. 41, pp. 823-830, 2005.

9. V. Andrieu, L. Praly, and A. Astolfi, "Homogeneous approximation, recursive obsertver and output feedback," SIAM Journal of Control and Optimization, vol. 47(4), pp. 1814-1850, 2008.

10. E. Cruz-Zavala, J. Moreno, and L. Fridman, "Uniform robust exact differentiator," IEEE Transactions on Automatic Control, vol. 56(11), pp. 2727-2733, 2011.

11. V. I. Zubov, Methods of A.M. Lyapunov and Their Applications. Noordhoff, Leiden, 1964.

12. A. Bacciotti and L. Rosier, Lyapunov Functions and Stability in Control Theory. Springer, 2005.

13. Y. Orlov, Discontinous systems: Lyapunov analysis and robust synthesis under uncertainty conditions. SpringerVerlag, 2009.

14. V. Korobov, "A general approach to synthesis problem," Doklady Academii Nauk SSSR, vol. 248, pp. 1051-1063, 1979.

15. J. Adamy and A. Flemming, "Soft variable-structure controls: a survey," Automatica, vol. 40, pp. 1821-1844, 2004.

16. H. Nakamura, N. Nakamura, and H. Nishitani, "Stabilization of homogeneous systems using implicit control lyhapunov functions," in 7th IFAC Symposium on Nonlinear Control Systems, 2007, pp. 561-566.

17. A. Polyakov, D. Efimov, and W. Perruquetti, "Finite-time stabilization using implicit lyapunov function technique,", in 9th Symposium on Nonlinear Control Systems, Toulouse, France, , 4-6 September 2013, pp. 140-145.

18. V. Zubov, "On systems of ordinary differential equations with generalized homogenous right-hand sides," Izvestia vuzov. Mathematica., vol. 1, pp. 80-88, 1958 (in Russian).

19. H. Hermes, "Nilpotent approximations of control systems and distributions," SIAM Journal of Control and Optimization, vol. 24, pp. 731-736, 1986.

20. L. Rosier, "Homogenous lyapunov function for homogenous continuous vector field," System \& Control Letters, vol. 19, pp. 467-473, 1992.

21. E. Bernuau, A. Polyakov, D. Efimov, and W. Perruquetti, "Verification of ISS, iISS and IOSS properties applying weighted homogeneity," System and Control Letters, vol. 62, no. 12, pp. 1159-1167, 2013.

22. A. Polyakov, D. Efimov, and W. Perruquetti, "Sliding mode control design for mimo systems: Implicit lyapunov function approach," in European Control Conference (ECC), 2014, pp. 2612-2617.

23. A. Levant, "On fixed and finite time stability in sliding mode control," in IEEE 52nd Conference on Decision and Control, 2013, pp. 4260-4265.

24. S. Drakunov, D. Izosimov, A. Lukyanov, V. Utkin, and V. Utkin, "Block control principle I," Automation and Remote Control, vol. 51(5), pp. 601-609, 1990.

25. A. Filippov, Differential equations with discontinuous right-hand sides. Kluwer, Dordrecht, 1988.

26. Y. Orlov, "Finite time stability and robust control synthesis of uncertain switched systems," SIAM Journal of Control and Optimization, vol. 43(4), pp. 1253-1271, 2005.

27. W. Hahn, Stability of Motion. New York: Springer-Verlag Berlin Heidelberg, 1967.

28. E. Bernuau, D. Efimov, W. Perruquetti, and A. Polyakov, "On an extension of homogeneity notion for differential inclusions," in European Control Conference, 2013, pp. 2204-2209.

29. H. Hermes, "Nilpotent and high-order approximations of vector field systems," SIAM Review, vol. 33, no. 2, pp. 238-264, 1991.

30. E. Ryan, "Universal stabilization of a class of nonlinear systems with homogeneous vector fields," Systems \& Control Letters, vol. 26, pp. 177-184, 1995.

31. Y. Hong, " $\mathrm{H}_{\infty}$ control, stabilization, and input-output stability of nonlinear systems with homogeneous properties," Automatica, vol. 37(7), pp. 819-829, 2001.

32. R. Courant and F. John, Introduction to calculus and analysis (Vol. II/1). New York: Springer, 2000.

33. A. Isidori, Nonlinear Control Systems. Springer-Verlag, N. Y. Inc., 1995.

34. F. H. Clarke, Y. S. Ledyaev, E. Sontag, and A. I. Subbotin, "Asymptotic controllability implies feedback stabilization," IEEE Transactions on Automatic Control, vol. 42, no. 10, pp. 1394-1407, 1997.

35. V. Utkin, J. Guldner, and J. Shi, Sliding Mode Control in Electro-Mechanical Systems. CRC Press., 2009. 